Discussion Paper Series A No.497

On Non-welfarist Social Ordering Functions

Naoki Yoshihara

September 2007

The Institute of Economic Research Hitotsubashi University

Kunitachi, Tokyo, 186-8603 Japan 


\title{
On Non-welfarist Social Ordering Functions ${ }^{\star}$
}

\author{
Naoki Yoshihara \\ Institute of Economic Research, Hitotsubashi University, Kunitachi, Japan \\ yosihara@ier.hit-u.ac.jp
}

Summary. In this paper, criticizing the welfarist's framework in traditional welfare economics which provides a rather limited perspective for social evaluation, we propose a more comprehensive framework in which extended social ordering functions (ESOFs) are introduced. In this framework, not only welfaristic values, but also non-welfaristic values can be treated appropriately. Then, we examine the possibility of non-welfarist ESOFs which meet a value of Individual Autonomy, a criterion of non-welfairst distributive justice, and the welfarist Pareto principle. First, there is no first best ESOF in the sense that the above three axioms are satisfied simultaneously. Second, however, we can show the existence of some second best ESOFs, using a weaker lexicographic application method.

JEL Classification Numbers: D63, I31, I38.

\section{Introduction}

Welfarism is defined as a methodology that evaluates social welfare according to the level of satisfaction with regard to individuals' subjective preferences. For this methodology, the criticisms by Amartya Sen [Sen (1979; 1980)], Ronald Dworkin [Dworkin (1981a; 2000)], and others are well known. These

\footnotetext{
* I express my special thanks to Kotaro Suzumura for his consistently kind and tolerant support as my supervisor since I was a Ph.D. student under him. I am also thankful to a referee of this paper for his many useful comments. A part of this paper was presented at the International Conference of Rational Choice, Individual Rights, and Non-welfaristic Normative Economics held in Hitotsubashi University, Tokyo, Japan, on March 11th-13th, 2006. Also, another part of this paper was presented at the Workshop on "Real Freedom for All" with Philippe van Parijs held in Ritsumeikan University, Kyoto, Japan, on July 7th, 2006. I am greatly thankful to all of the participants in these meetings, in particular, N. Baigent, K. Basu, M. Fluerbaey, P. Hammond, Y. Sprumont, K. Tadenuma, P. van Parijs, and Y. Xu for their comments. It is worth noting that the main body of this paper is deeply indebted to the joint work with R. Gotoh and K. Suzumura in Gotoh, Suzumura, and Yoshihara (2005).
} 
criticized the limited scope of information used to evaluate social welfare in the aforementioned methodology. Moreover, they criticize the welfarist neutral attitude vis-à-vis the problem of what types of preferences are satisfied. There are types of preferences, such as the utility of individual offensive tastes, that of expensive tastes, that of formation of the adaptive preference, or that of cheaper tastes such as in the case of the 'termed housewife,' all of which should be carefully and distinctively treated in the evaluation of social welfare from an ethical point of view. The point of these critiques is that the welfarist evaluation has no concern for such preferential differences.

The problems with welfarist methodology can emerge in a more acute form within the arguments of welfare economics such as the hypothetical compensation principle. For instance, let us take the Kaldor principle, which declares an alternative $x$ to be superior to an alternative $y$ if and only if there is another alternative $z$ which is reached through redistribution from $x$ such that $z$ is better than $y$ according to the Pareto principle. This principle is welfarist in nature, since it evaluates policies based only on satisfaction of individuals' preferences of goods and services, and it is also an extension of the Pareto principle as is clear from its definition. It makes clear judgments on policy changes, based on whether or not there is a possibility of potential Pareto improvement of said changes. It is also well-known that the validity of executing a policy according to the hypothetical compensation principle is possibly confirmed by means of such monetary measures as the gross national income test, whenever the change in resource allocation caused by the policy is not radical.

According to the above argument, the notion of social welfare that the hypothetical compensation principle considers as its premise is no less than the sum of subjective satisfaction levels obtained from the consumption of 'marketable' goods and services, which can be evaluated by monetary measures. However, the notion of social welfare in general is broad enough to encompass a wide range of ethical viewpoints. The social welfare that welfarist's welfare economics refers to is not as broad, but it is limited to (market) economic welfare.

Against such an argument, the following objection may arise from the welfarist position:

It is true that the social welfare analysis utilized in conventional applied economics concerns only the social welfare as the total sum of satisfaction of individuals' preferences over goods and services, which is convertible to monetary value. However, the concept of social welfare can be extended so as to consider the 'utility' from an outcome other than the private consumption of goods and services, extending the domain of the individual utility function if necessary.

Such an approach is one way to expand the limited informational basis of conventional welfarist's welfare economics. However, even using this approach, we would not be able to avoid the above mentioned criticisms of Sen and Dworkin, 
because it treats and evaluates everything, including the private consumption of goods and services as well as intrinsic goods such as friendship, through the prism of the same subjective utility functions. The concept of social welfare in this approach is still corresponding solely to the satisfaction of individual subjective preferences. In contrast, this paper argues that the social welfare should be evaluated, not only from the perspective of subjective preferences or tastes, but also from the perspective of welfare and well-being that cannot be grasped by utilizing such preferences. For instance, the viewpoint of "respect for liberal rights" presented in Sen's Liberal Paradox [Sen (1970a,b)] and his theory of "functioning and capability" [Sen (1985)] offer such concepts of welfare and well-being.

The criticism of welfarism mentioned above becomes relevant not only in the discussion of the criteria of policy evaluation based on the hypothetical compensation principle, but also in the general discussion of welfarist social welfare functions. A social welfare function associates an ordering over social alternatives with each social choice environment. According to the ordering derived from such a function, the society can identify what the most desired policies are, which would realize the most desired social alternatives.

The basic problem in this context is what type of social welfare function should be constructed, and it is in the course of such discussions that the conventional Bergson-Samuelson (B-S) social welfare functions are perceived as problematic. The very reason for this is that in the B-S social welfare functions, the level of individual satisfaction with their subjective preferences is the sole basis of information. However, the orderings over social alternatives given by social welfare functions should reflect an adequate indicator of individuals' well-being. The criticism of welfarism mentioned so far indicates that individual satisfaction with their subjective preferences is no more than one aspect of welfare and therefore a more pluralistic viewpoint is necessary.

To treat such a pluralistic viewpoint appropriately, a more comprehensive framework is necessary. As such, we propose the extended framework within which not only welfarist notions of individual well-being, but also non-welfarist notions of consequential values, as well as non-consequential values, can be taken into consideration. The extended framework in this paper takes a pair of feasible allocation and allocation rule as an informational basis for the social evaluation of economic policies, and it also proposes to make use of extended social ordering functions, each of which associates a social ordering over the set of pairs of feasible allocations and allocation rules with each economic environment. Within such an extended framework, we propose three basic criteria, each of which respectively represents: 1) a value of individual autonomy, 2) a value of non-welfaristic consequentialism, and 3) a value of welfarist consequentialism. Moreover, we examine the possibility of extended social ordering functions which satisfy these three pluralistic values.

There has been recently some literature such as Kaplow and Shavell (2001) and Blackorby, Bossert, and Donaldson (2005) which also discuss some sorts of 'extended' social ordering functions satisfying some pluralistic values. In 
their frameworks of social ordering functions, not only the profile of utility information, but also the profile of non-welfaristic information are taken into account. Then, both papers show that even in such frameworks with nonwelfaristic information, the feasible class of social ordering functions is reduced to that of the welfarist types only, whenever the Pareto principle is required. Despite the conclusions of Kaplow and Shavell (2001) and Blackorby et al. (2005), we show in this paper that it is possible to construct a desirable social ordering function that has the properties of the welfarist Pareto principle and the non-welfarist criteria. There is no contradiction between the results of these papers and ours, as discussed in Section 5 of this paper.

Section 2 introduces the basic framework and the basic three axioms. Section 3 discusses a fundamental incompatibility of these three axioms, and section 4 explores the possibility of second best extended social ordering functions. Section 5 gives some remarks on the related literature such as Kaplow and Shavell (2001) and Blackorby et al. (2005).

\section{Beyond the welfarist limitation}

The need for the pluralistic approach was argued by John Rawls [Rawls (1971)], Amartya Sen [Sen (1980); 1985]], and Philippe Van Parijs [Van Parijs $(1992 ; 1993 ; 1995)]$. Based on the normative theories of these three nonwelfarists, we propose three basic criteria.

The first criterion is that individual autonomy in contemporary society should be guaranteed. It is a liberal value that contemporary civil societies respect as an important aspect for evaluating individual well-being. In fact, as opposed to the feudal society and the centralized socialist society where individual autonomy is suppressed, the modern civil society might be characterized as having a certain level of political liberalism in legal systems, a certain level of freedom of choice both in political and economic decision-makings, and a certain level of decentralized decision-making mechanisms such as markets, all of which constitute a necessary condition for the guarantee of individual autonomy. Such a viewpoint would suggest a certain constraint over the class of 'desired' social ordering functions. That is to say, if the social economic system cannot guarantee the decentralization and the freedom of choice in decision-making, the welfare that individuals receive under such social situations will not be highly valued by 'desired' social ordering functions, even if the system may support a sufficient level of individual consumption. Thus, this criterion represents a non-consequential value in nature.

The second criterion is that each and every individual should have as much opportunity to do whatever he might want to do as is feasibly possible. This criterion represents a non-welfaristic consequential value in the sense of the following two points: First, although this criterion pertains to social outcomes in terms of individual well-being, it hinges on an objective notion of individual well-being as opposed to welfarist criteria. Second, this criterion does not 
concern the realization of individual well-being itself, but rather it pertains to the opportunity to pursue or realize individual well-being. Given these points, theories of distributive justice are relevant in the discussion of what concept of individual well-being is appropriate, and of what types of equity notions should be applied to the assignment problem of individual opportunity sets.

The third criterion represents a well-known welfarist consequential value such as the Pareto principle. It is worth noting that the standpoint of nonwelfarism does not exclude welfarist notions of well-being. I believe that satisfaction of individual subjective preferences is still an important component of the informational basis used to constitute an overall notion of individual well-being. Thus, the Pareto principle is also taken into consideration as a condition imposed on 'desired' social ordering functions.

With this discussion in mind, the question that arises here is whether it is possible to construct a social ordering function consistent with the different pluralistic criteria mentioned above.

\subsection{A Framework of Extended Social Ordering Functions}

On the basis of the problems propounded in the previous section, in the following subsection, the notion of extended social ordering function is introduced, which is based on the proposal of Gotoh, Suzumura, and Yoshihara (2005). There are two goods, one of which is an input (labor time) $x \in \mathbb{R}_{+}$to be used to produce the other good $y \in \mathbb{R}_{+}{ }^{1}$ There is a set $N=\{1, \ldots, n\}$ of agents, where $2 \leq n<+\infty$. Each agent $i^{\prime}$ s consumption is denoted by $z_{i}=\left(x_{i}, y_{i}\right)$, where $x_{i}$ denotes his labor time, and $y_{i}$ the amount of his output. All agents face a common upper bound of labor time $\bar{x}$, where $0<\bar{x}<+\infty$, and so have the same consumption set $Z \equiv[0, \bar{x}] \times \mathbb{R}_{+}$.

Each agent $i^{\prime}$ s preference is defined on $Z$ and represented by a utility function $u_{i}: Z \rightarrow \mathbb{R}$, which is continuous and quasi-concave on $Z$, strictly monotonic (decreasing in labor time and increasing in the share of output) on $\stackrel{\circ}{Z} \equiv[0, \bar{x}) \times \mathbb{R}_{++},{ }^{2}$ and $u_{i}(x, 0)=0$ for any $x \in[0, \bar{x}]$. We use $\mathcal{U}$ to denote the class of such utility functions.

Each agent $i$ has a labor skill, $s_{i} \in \mathbb{R}_{+}$. The universal set of skills for all agents is denoted by $\mathcal{S}=\mathbb{R}_{+}$. The skill $s_{i} \in \mathcal{S}$ is $i^{\prime}$ s effective labor supply per hour measured in efficiency units. It can also be interpreted as $i^{\prime}$ s labor intensity exercised in production. Thus, if the agent's labor time is $x_{i} \in[0, \bar{x}]$ and his skill is $s_{i} \in \mathcal{S}$, then $s_{i} x_{i} \in \mathbb{R}_{+}$denotes the agent's effective labor contribution to production measured in efficiency units. The production technology is a function $f: \mathbb{R}_{+} \rightarrow \mathbb{R}_{+}$, that is continuous, strictly increasing, concave, and $f(0)=0$. For simplicity, we fix $f$. Thus, an economy is a pair of profiles $\mathbf{e} \equiv(\mathbf{u}, \mathbf{s})$ with $\mathbf{u}=\left(u_{i}\right)_{i \in N} \in \mathcal{U}^{n}$ and $\mathbf{s}=\left(s_{i}\right)_{i \in N} \in \mathcal{S}^{n}$. Denote the class of such economies by $\mathcal{E} \equiv \mathcal{U}^{n} \times \mathcal{S}^{n}$.

\footnotetext{
${ }^{1}$ The symbol $\mathbb{R}_{+}$denotes the set of non-negative real numbers.

${ }^{2}$ The symbol $\mathbb{R}_{++}$denotes the set of positive real numbers.
} 
Given $\mathbf{s}=\left(s_{i}\right)_{i \in N} \in \mathcal{S}^{n}$, an allocation $\mathbf{z}=\left(x_{i}, y_{i}\right)_{i \in N} \in Z^{n}$ is feasible for $\mathbf{s}$ if $\sum y_{i} \leq f\left(\sum s_{i} x_{i}\right)$. We denote by $Z(\mathbf{s})$ the set of feasible allocations for $\mathbf{s} \in \mathcal{S}^{n}$. An allocation $\mathbf{z}=\left(z_{i}\right)_{i \in N} \in Z^{n}$ is Pareto efficient for $\mathbf{e}=(\mathbf{u}, \mathbf{s}) \in \mathcal{E}$ if $\mathbf{z} \in Z(\mathbf{s})$ and there does not exist $\mathbf{z}^{\prime}=\left(z_{i}^{\prime}\right)_{i \in N} \in Z(\mathbf{s})$ such that for all $i \in N, u_{i}\left(z_{i}^{\prime}\right) \geq u_{i}\left(z_{i}\right)$, and for some $i \in N, u_{i}\left(z_{i}^{\prime}\right)>u_{i}\left(z_{i}\right)$. We use $P(\mathbf{e})$ to denote the set of Pareto efficient allocations for $\mathbf{e} \in \mathcal{E}$.

To complete the description of how our economy functions, what remains is to specify an allocation rule which assigns, to each $i \in N$, how many hours he/she works, and how much share of output he/she receives in return. In this paper, an allocation rule is a game form which is a pair $\gamma=(M, g)$, where $M=M_{1} \times \cdots \times M_{n}$ is the set of admissible profiles of individual strategies, and $g$ is the outcome function which maps each strategy profile $\mathbf{m} \in M$ into a unique outcome $g(\mathbf{m}) \in Z^{n}$. For each $\mathbf{m} \in M, g(\mathbf{m})=\left(g_{i}(\mathbf{m})\right)_{i \in N}$, where $g_{i}(\mathbf{m})=\left(g_{i 1}(\mathbf{m}), g_{i 2}(\mathbf{m})\right)$ and $g_{i 1}(\mathbf{m}) \in[0, \bar{x}]$ and $g_{i 2}(\mathbf{m}) \in \mathbb{R}_{+}$for each $i \in N .^{3}$ Let $\Gamma$ be the set of all possible such allocation rules. Given $\gamma=$ $(M, g) \in \Gamma$ and $\mathbf{e} \in \mathcal{E}$, a non-cooperative game $(\gamma, \mathbf{e}) \in \Gamma \times \mathcal{E}$ is obtained.

Throughout this paper, we will focus on the Nash equilibrium concept in our analysis of the performance of game forms as allocation rules. Given $\gamma=(M, g)$, let $\mathbf{m}_{-i}=\left(m_{1}, \ldots, m_{i-1}, m_{i+1}, \ldots, m_{n}\right) \in M_{-i} \equiv \times_{j \in N \backslash\{i\}} M_{j}$ for each $\mathbf{m} \in M$ and $i \in N$. Given an $\mathbf{m}_{-i} \in M_{-i}$ and an $m_{i}^{\prime} \in M_{i},\left(m_{i}^{\prime} ; \mathbf{m}_{-i}\right)$ is an admissible strategy profile obtained from $\mathbf{m}$ by replacing $m_{i}$ with $m_{i}^{\prime}$. Given a game $(\gamma, \mathbf{e}) \in \Gamma \times \mathcal{E}, \mathbf{m}^{*} \in M$ is a (pure strategy) Nash equilibrium if $u_{i}\left(g_{i}\left(\mathbf{m}^{*}\right)\right) \geq u_{i}\left(g_{i}\left(m_{i}, \mathbf{m}_{-i}^{*}\right)\right)$ for each $i \in N$ and each $m_{i} \in M_{i}$. The set of all Nash equilibria of $(\gamma, \mathbf{e})$ is denoted by $N E(\gamma, \mathbf{e})$. A feasible allocation $\mathbf{z}^{*} \in$ $Z(\mathbf{s})$ is a (pure strategy) Nash equilibrium allocation of $(\gamma, \mathbf{e})$ if $\mathbf{z}^{*}=g\left(\mathbf{m}^{*}\right)$ for some $\mathbf{m}^{*} \in N E(\gamma, \mathbf{u})$. The set of all Nash equilibrium allocations of $(\gamma, \mathbf{e})$ is denoted by $\tau(\gamma, \mathbf{e})$.

The domain of social preference relations in this paper is given by pairs of allocations and allocation rules as game forms, which we call extended social alternatives. The intended interpretation of an extended social alternative, viz., a pair $(\mathbf{z}, \gamma) \in Z^{n} \times \Gamma$, is that an allocation $\mathbf{z}$ is attained through an allocation rule $\gamma^{4}$ Moreover, given $\mathbf{e} \in \mathcal{E}$, an extended social alternative $(\mathbf{z}, \gamma) \in Z^{n} \times \Gamma$ is realizable if $\mathbf{z} \in Z(\mathbf{s}) \cap \tau(\gamma, \mathbf{e})$. Let $\mathcal{R}(\mathbf{e})$ denote the set of realizable extended social alternatives under $\mathbf{e} \in \mathcal{E}$.

What we call an extended social ordering function (ESOF) is a mapping $Q: \mathcal{E} \rightarrow\left(Z^{n} \times \Gamma\right)^{2}$ such that $Q(\mathbf{e})$ is an ordering on $\mathcal{R}(\mathbf{e})$ for every $\mathbf{e} \in \mathcal{E} .^{5}$ The intended interpretation of $Q(\mathbf{e})$ is that, for any $\left(\mathbf{z}^{1}, \gamma^{1}\right),\left(\mathbf{z}^{2}, \gamma^{2}\right) \in \mathcal{R}(\mathbf{e})$, $\left(\left(\mathbf{z}^{1}, \gamma^{1}\right),\left(\mathbf{z}^{2}, \gamma^{2}\right)\right) \in Q(\mathbf{e})$ holds if and only if realizing a feasible allocation

\footnotetext{
${ }^{3}$ Note that $g_{i 1}\left(m_{i}, \mathbf{m}_{-i}\right)=x_{i}$ is describing the work-hour supply of an individual $i \in N$ that the outcome function designates corresponding to the strategy $\left(m_{i}, \mathbf{m}_{-i}\right) \in M$.

4 The concept of an extended social alternative was introduced by Pattanaik and Suzumura (1996), capitalizing on the suggestion by Arrow (1951, pp.89-91).

${ }^{5}$ A binary relation $R$ on a universal set $X$ is a quasi-ordering if it satisfies reflexivity and transitivity. An ordering is a quasi-ordering satisfying completeness as well.
} 
$\mathbf{z}^{1}$ through an allocation rule $\gamma^{1}$ is at least as good as realizing a feasible allocation $\mathbf{z}^{2}$ through an allocation rule $\gamma^{2}$ according to the social judgments embodied in $Q(\mathbf{e})$. The asymmetric part and the symmetric part of $Q(\mathbf{e})$ will be denoted by $P(Q(\mathbf{e}))$ and $I(Q(\mathbf{e}))$, respectively. The set of all ESOFs will be denoted by $\mathcal{Q}$.

The notion of extended social ordering functions enables us to treat the criteria of individual autonomy, equitable assignment of opportunities in terms of objective well-being, and the Pareto principle in a unified framework. Within the domain $Z^{n} \times \Gamma$ of social preference orderings derived from ESOFs, the component of game forms constitutes necessary data for formulating orderings based on the criterion of individual autonomy, whereas the data of feasible allocations is relevant to the remaining two criteria.

In the following part, the above mentioned three criteria are formalized as axioms applicable to ESOFs.

\section{(I) Individual Autonomy in terms of Choice of Labor Hours}

According to the theory of individual liberty that John Stuart Mill proposed [Mill (1859)], there ought to exist in human life a certain minimal sphere of personal liberty that should not be interfered with by anybody other than the person in question. Such a sphere should be socially respected and protected as part of individual rights in a liberal society. The question where exactly to draw the boundary between the sphere of personal liberty and that of social authority is a matter of great dispute, and, indeed, how large of a sphere each individual should be entitled to is a controversial issue. Nevertheless, the notion of the inviolability of a minimal sphere of individual liberal rights seems to be deeply ingrained in our social and political fabric.

Thus, a resource allocation policy would rarely be accepted, if its goal or its implementation were incompatible with this minimal guarantee of individual liberty. Such a viewpoint is relevant to our first axiom of extended social ordering functions. We will discuss what constitutes the minimal guarantee of individual rights in the context of resource allocations that this paper considers.

In cases of resource allocation problems, the components of political freedom and the non-economic aspects of individual rights might be assumed to be already established. However, there still remain non-established economic parts of individual rights, which might be either treated as parameters or as variables for relevant resource allocation problems. For instance, we may view self-ownership as such a right guaranteeing individual autonomy. The notion of self-ownership originates from the argument of the Lockean proviso of John Locke and was used by Robert Nozick (1974) as the principle to justify private ownership in capitalist societies. Nevertheless, the notion of self-ownership can be connected with two versions of entitlement principles, that is, the entitlement principles in the weak sense and in the strong sense, as van Parijs (1995) discussed. 
The entitlement principle in the weaker sense regards self-ownership as a variable for society. Thus, according to this weaker sense of the principle, self-ownership can be seen as freedom or respect for the decision-making of individuals and identified with political freedom and freedom of choice of occupations, etc. ${ }^{6}$ In this version, the notion of self-ownership is entirely consistent with redistribution policies which may induce the reconstruction of a given rights structure in order to achieve a given distributional goal. This is actually the position that van Parijs (1995) takes. On the other hand, the entitlement principle in the stronger sense no longer views self-ownership as a control variable, but as a parameter which society respects. This stronger sense of the principle can be identified with the arguments made by John Locke. This principle also made a solid basis for the original appropriation of unowned external resources, which was proposed by Libertarians including Locke and Nozick.

We also take the same position as van Parijs (1995) regarding the notion of self-ownership, and identify the contents of individual liberal rights within the context of resource allocation problems. First, individual liberal rights guarantee freedom of choice in terms of personal consumption. That is, other than the individual in question, no one else has the right to decide the way to dispose of private goods and leisure time available to him/her. W. l.o.g., we should assume that the right of freedom of choice in consumption, in the context of passive freedom, is presumed to be guaranteed in standard economic models of resource allocation problems.

Secondly, individual liberal rights contain the right to freedom from forced labor. This right consists of the freedom to choose a profession, the freedom to enter into an employment contract, etc. However, in simple economic models like this paper, this right may be reduced to the right to choose labor hours, because there is no difference in profession, and all individuals engage in homogeneous labor.

The right to choose labor hours is defined as follows:

Definition 1 [Kranich (1994)]: An allocation rule $\gamma=(M, g) \in \Gamma$ is laborsovereign if, for all $i \in N$ and all $x_{i} \in[0, \bar{x}]$, there exists $m_{i} \in M_{i}$ such that, for all $\mathbf{m}_{-i} \in M_{-i}, \quad g_{i 1}\left(m_{i}, \mathbf{m}_{-i}\right)=x_{i}$.

Let $\Gamma_{L}$ denote the subclass of $\Gamma$ which consists solely of allocation rules satisfying labor sovereignty. Then:

Labor Sovereignty (LS): For any $\mathbf{e} \in \mathcal{E}$ and any $(\mathbf{z}, \gamma),\left(\mathbf{z}^{\prime}, \gamma^{\prime}\right) \in \mathcal{R}(\mathbf{e})$, if $\gamma \in \Gamma_{L}$ and $\gamma^{\prime} \in \Gamma \backslash \Gamma_{L}$, then $\left((\mathbf{z}, \gamma),\left(\mathbf{z}^{\prime}, \gamma^{\prime}\right)\right) \in P(Q(\mathbf{e}))$.

\footnotetext{
${ }^{6}$ In fact, van Parijs (1995) insists, "Though not strictly equivalent to 'basic liberties' or 'human rights' as expressed, for example, in Rawls's first principle of justice or in the constitutions of liberal democracies, self-ownership is closely associated with most of them." [van Parijs (1995; p. 235; NOTES Chapter 1, 8.)]
} 
The axiom LS manifests that the extended social alternative with labor sovereign rule should be given a higher priority than any alternative without it. This manifestation should be implemented regardless of what resource allocations the labor sovereign rule or the non-labor sovereign rule realizes as Nash equilibrium outcomes. This expresses an extremely non-consequential value.

Note that if a society executes a non-labor sovereign rule, then such a society might allow the policy-maker to execute some sort of forced labor. The axiom LS rejects such a society and an economic institution. According to this axiom, even an egalitarian redistribution policy would not be accepted unless it were implemented without using forced labor. I believe that the principle of self-ownership based on the weak sense of entitlement principle, and also, even Rawls's first principle of justice [Rawls (1971)] should have the form of $\mathbf{L S}$ within this economic model.

\section{(II) Evaluation based on a criterion of Distributive Justice}

Our next criterion is meant to capture an aspect of non-welfaristic egalitarianism. It hinges on what theories of distributive justice we take, which requires an instrument that incorporates the various criteria of distributive justice.

Such an instrument is given by a mapping $J: \mathcal{E} \rightarrow Z^{n} \times Z^{n}$ which associates a binary relation $J(\mathbf{e}) \subseteq Z(\mathbf{s}) \times Z(\mathbf{s})$ with each economy e $\in \mathcal{E}$. Denote the class of binary relation mappings by $\mathcal{J}$. Such a binary relation $J$ (e) represents a criterion based on a certain theory of distributive justice and alternative feasible allocations are ranked according to this criterion. For instance, if the mapping $J$ represents Sen's theory of equality of capability, then $J(\mathbf{e})$ provides a ranking over alternative capability assignments available to each economy $\mathbf{e} \in \mathcal{E}$, and the rational choice set, derived from this $J(\mathbf{e})$, is regarded as consisting of the most 'equitable' capability assignments under $\mathbf{e} \in \mathcal{E}{ }^{7}$ In this case, the ranking made by $J$ should be invariant with respect to the change in the profile of utility functions: that is, $J(\mathbf{e})=J\left(\mathbf{e}^{\prime}\right)$ holds whenever $\mathbf{s}=\mathbf{s}^{\prime}$ holds. In contrast, if $J$ represents Dworkinian theory of "equality of resources" [Dworkin, $(1981 b ; 2000)], J$ might not have such an invariance property: that is, $J(\mathbf{e}) \neq J\left(\mathbf{e}^{\prime}\right)$ may hold even if $\mathbf{s}=\mathbf{s}^{\prime}$. Moreover, if $J$ represents the theory of "equality of welfare," then $J(\mathbf{e})=J\left(\mathbf{e}^{\prime}\right)$ should hold for any $\mathbf{e}, \mathbf{e}^{\prime} \in \mathcal{E}$ with $\mathbf{u}=\mathbf{u}^{\prime}$. In such a way, this mapping can be universally applicable. Moreover, if $J$ represents the criterion of leximin assignment of opportunity sets suggested by van Parijs (1995), then $J$ should rationalize feasible allocations satisfying undominated diversity [van Parijs (1995)]. ${ }^{8}$ In

\footnotetext{
${ }^{7}$ Gotoh, Suzumura, and Yoshihara (2005) argues this case.

${ }^{8}$ Given any economy $\mathbf{e}=(\mathbf{u}, \mathbf{s}) \in \mathcal{E}$, a feasible allocation $\mathbf{z} \in Z(\mathbf{s})$ meets undominated diversity if for any $i, j \in N$, there exists at least one individual $k \in N$ such that $u_{k}\left(z_{i}\right) \geq u_{k}\left(z_{j}\right)$.
} 
any case, if $J$ represents a criterion of distributive justice, it should satisfy at least the following requirement:

Minimal Egalitarianism (ME): For each $\mathbf{e}=(\mathbf{u}, \mathbf{s}) \in \mathcal{E}$ and each $\mathbf{z}, \mathbf{z}^{\prime} \in$ $Z$ (s) such that for any $i, j \in N, s_{i}=s_{j}$ and $x_{i}=x_{j}=x_{i}^{\prime}=x_{j}^{\prime}$, if there exist $i, j \in N$ such that $y_{i}^{\prime}>y_{i} \geq y_{j}>y_{j}^{\prime}$ and $y_{k}=y_{k}^{\prime}$ for any $k \neq i, j$, then $\left(\mathbf{z}, \mathbf{z}^{\prime}\right) \in P(J(\mathbf{e}))$.

Denote the class of binary relation mappings satisfying ME by $\mathcal{J}^{M E}$. This axiom says that, any transfer from a poorer person to a richer person, given that these two persons provide the same labor hours and other things remain the same, should be undesirable, which shares the same spirit as the PigouDalton principle. Note that various types of distributive justice satisfy ME if each of those theories is formulated as a particular $J \in \mathcal{J}$. In fact, Sen's theory of equality of capability, Dworkin's theory of equality of resources, van Parijs's undominated diversity, and even the equity as no-envy [Foley (1967)] respectively, could have their own representations within $\mathcal{J}^{M E}$.

If $J \in \mathcal{J}^{M E}$ represents a non-welfarist egalitarianism with objective wellbeing indices, then $J$ might satisfy the following requirement:

Objective Egalitarianism (OE): For each $\mathbf{e}=(\mathbf{u}, \mathbf{s}), \mathbf{e}^{\prime}=\left(\mathbf{u}^{\prime}, \mathbf{s}^{\prime}\right) \in \mathcal{E}$, if $\mathbf{s}=\mathbf{s}^{\prime}$, then $J(\mathbf{e})=J\left(\mathbf{e}^{\prime}\right)$.

Denote the class of mappings which satisfy $\mathbf{M E}$ and $\mathbf{O E}$ by $\mathcal{J}^{M O E}$. Note that any $J \in \mathcal{J}^{M O E}$ is invariant with respect to change in the profile of individual utility functions. Thus, for instance, Sen's theory of equality of capability has its representation within $\mathcal{J}^{M O E}$, as formulated in Gotoh, Suzumura, and Yoshihara (2005). In contrast, the representation of van Parijs's undominated diversity does not belong to $\mathcal{J}^{M O E}$, since undominated diversity needs information about individual utility functions.

Now, our second axiom on ESOFs is given by means of the binary relation mapping $J \in \mathcal{J}$, as follows:

Respect for $J$-based fairness (J-RF): For any $\mathbf{e} \in \mathcal{E}$ and any $(\mathbf{z}, \gamma),\left(\mathbf{z}^{\prime}, \gamma^{\prime}\right) \in$ $\mathcal{R}(\mathbf{e})$, if $\mathbf{z}=(\mathbf{x}, \mathbf{y}), \mathbf{z}^{\prime}=\left(\mathbf{x}^{\prime}, \mathbf{y}^{\prime}\right)$ and $\mathbf{x}=\mathbf{x}^{\prime}$, then:

$$
\begin{aligned}
& \left((\mathbf{z}, \gamma),\left(\mathbf{z}^{\prime}, \gamma^{\prime}\right)\right) \in Q(\mathbf{e}) \Leftrightarrow\left(\mathbf{z}, \mathbf{z}^{\prime}\right) \in J(\mathbf{e}) ; \\
& \left((\mathbf{z}, \gamma),\left(\mathbf{z}^{\prime}, \gamma^{\prime}\right)\right) \in P(Q(\mathbf{e})) \Leftrightarrow\left(\mathbf{z}, \mathbf{z}^{\prime}\right) \in P(J(\mathbf{e})) .
\end{aligned}
$$

In evaluating the relative wellness of any two extended alternatives, the axiom J-RF focuses only on the corresponding feasible allocations, and under a certain constraint, it claims that the evaluation by the ESOF over extended alternatives should be consistent with the evaluation by $J$ over the corresponding feasible allocations. Here, the "certain constraint" is given by $" \mathbf{z}=(\mathbf{x}, \mathbf{y}), \mathbf{z}^{\prime}=\left(\mathbf{x}^{\prime}, \mathbf{y}^{\prime}\right)$ and $\mathbf{x}=\mathbf{x}^{\prime} . "$ 
It may well be asked why $J$-RF imposes the premise $\mathbf{x}=\mathbf{x}^{\prime}$. The reasons are twofold. First, the choice of individual labor hours is a matter to be left to individual responsibility, and social value judgements should respect individual choices accordingly. Second, if the requirement of $J$-RF is applied to ESOFs without the premise $\mathbf{x}=\mathbf{x}^{\prime}$, this instantly causes the incompatibility with the Paretian axiom, which will be discussed below.

As such, J-RF evaluates the desirability of extended social alternatives only from the viewpoint of $J$-fairness on resource allocations. Unlike the axiom $\mathbf{L S}, \mathbf{J}-\mathbf{R F}$ represents a consequentialist value. This is because this axiom evaluates the extended alternatives based solely on the evaluation of their corresponding resource allocations.

\section{(III) Evaluation based on the welfarist value}

Finally, let us introduce the axiom of ESOFs based on the welfarist value. It is defined as an extension of the standard Pareto principle:

Pareto in Allocations (PA): For any $\mathbf{e} \in \mathcal{E}$ and any $(\mathbf{z}, \gamma),\left(\mathbf{z}^{\prime}, \gamma^{\prime}\right) \in \mathcal{R}(\mathbf{e})$, if $u_{i}\left(z_{i}\right)>u_{i}\left(z_{i}^{\prime}\right)$ for all $i \in N$, then $\left((\mathbf{z}, \gamma),\left(\mathbf{z}^{\prime}, \gamma^{\prime}\right)\right) \in P(Q(\mathbf{e}))$, and if $u_{i}\left(z_{i}\right)=u_{i}\left(z_{i}^{\prime}\right)$ for all $i \in N$, then $\left((\mathbf{z}, \gamma),\left(\mathbf{z}^{\prime}, \gamma^{\prime}\right)\right) \in I(Q(\mathbf{e}))$.

The axiom PA also focuses only on the feasible allocation in evaluating the relative wellness of any two extended alternatives, and it claims that the evaluation by the ESOF over extended alternatives should be consistent with the Pareto superiority relation or the Pareto indifference relation over feasible allocations. Thus, by the almost same reason as the case of J-RF, PA also represents a position of consequentialism.

\section{Impossibility of ESOFs satisfying LS, J-RF, and PA}

Now, we are ready to discuss the existence of ESOFs which simultaneously satisfy the axioms LS, J-RF, and PA. According to the technique introduced in Appendix 1 of this paper, we can see this problem by examining the properties of binary relation functions, each of which respectively represents one of the above mentioned axioms.

Let $Q^{L}: \mathcal{E} \rightarrow\left(Z^{n} \times \Gamma\right)^{2}$ be a binary relation function such that for any $\mathbf{e} \in \mathcal{E}$ and any $(\mathbf{z}, \gamma),\left(\mathbf{z}^{\prime}, \gamma^{\prime}\right) \in \mathcal{R}(\mathbf{e})$, the following holds:

$$
\begin{aligned}
& \left((\mathbf{z}, \gamma),\left(\mathbf{z}^{\prime}, \gamma^{\prime}\right)\right) \in P\left(Q^{L}(\mathbf{e})\right) \Leftrightarrow\left[\gamma \in \Gamma_{L} \& \gamma^{\prime} \notin \Gamma_{L}\right] \\
& \left((\mathbf{z}, \gamma),\left(\mathbf{z}^{\prime}, \gamma^{\prime}\right)\right) \in I\left(Q^{L}(\mathbf{e})\right) \Leftrightarrow(\mathbf{z}, \gamma)=\left(\mathbf{z}^{\prime}, \gamma^{\prime}\right) .
\end{aligned}
$$

Let $Q^{J F}: \mathcal{E} \rightarrow\left(Z^{n} \times \Gamma\right)^{2}$ be a binary relation function such that for any $\mathbf{e} \in \mathcal{E}$ and any $(\mathbf{z}, \gamma),\left(\mathbf{z}^{\prime}, \gamma^{\prime}\right) \in \mathcal{R}(\mathbf{e})$ with $\mathbf{z}=(\mathbf{x}, \mathbf{y})$ and $\mathbf{z}^{\prime}=\left(\mathbf{x}^{\prime}, \mathbf{y}^{\prime}\right)$, the following holds: 


$$
\begin{aligned}
& \left((\mathbf{z}, \gamma),\left(\mathbf{z}^{\prime}, \gamma^{\prime}\right)\right) \in I\left(Q^{J F}(\mathbf{e})\right) \Leftrightarrow \mathbf{x}=\mathbf{x}^{\prime} \text { and }\left(\mathbf{z}, \mathbf{z}^{\prime}\right) \in I(J(\mathbf{e})) \\
& \left((\mathbf{z}, \gamma),\left(\mathbf{z}^{\prime}, \gamma^{\prime}\right)\right) \in P\left(Q^{J F}(\mathbf{e})\right) \Leftrightarrow \mathbf{x}=\mathbf{x}^{\prime} \text { and }\left(\mathbf{z}, \mathbf{z}^{\prime}\right) \in P(J(\mathbf{e})) .
\end{aligned}
$$

Let $Q^{P A}: \mathcal{E} \rightarrow\left(Z^{n} \times \Gamma\right)^{2}$ be a binary relation function such that for any $\mathbf{e} \in \mathcal{E}$ and any $(\mathbf{z}, \gamma),\left(\mathbf{z}^{\prime}, \gamma^{\prime}\right) \in \mathcal{R}(\mathbf{e})$, the following holds:

$$
\begin{aligned}
& \left((\mathbf{z}, \gamma),\left(\mathbf{z}^{\prime}, \gamma^{\prime}\right)\right) \in P\left(Q^{P A}(\mathbf{e})\right) \Leftrightarrow u_{i}\left(z_{i}\right)>u_{i}\left(z_{i}^{\prime}\right) \quad(\forall i \in N) \\
& \left((\mathbf{z}, \gamma),\left(\mathbf{z}^{\prime}, \gamma^{\prime}\right)\right) \in I\left(Q^{P A}(\mathbf{e})\right) \Leftrightarrow u_{i}\left(z_{i}\right)=u_{i}\left(z_{i}^{\prime}\right) \quad(\forall i \in N) .
\end{aligned}
$$

Let a binary relation function $Q$ be called the minimal relation function w.r.t. an axiom $a$ if $Q$ satisfies the axiom $a$, and for any binary relation function $Q^{\prime}$ satisfying the axiom $a, Q^{\prime}(\mathbf{e}) \supseteq Q(\mathbf{e})$ holds. Note that each of $Q^{L}, Q^{J F}$, and $Q^{P A}$ is the minimal relation function w.r.t. each of the axioms $\mathbf{L S}, \mathbf{J}-\mathbf{R F}$, and PA.

Thus, if there exists an ESOF $Q$ which satisfies these three axioms, $Q(\mathbf{e}) \supseteq$ $Q^{L}(\mathbf{e}) \cup Q^{J F}(\mathbf{e}) \cup Q^{P A}(\mathbf{e})$ holds for each $\mathbf{e} \in \mathcal{E}$. Define $Q^{L J P}$ by $Q^{L J P}(\mathbf{e}) \equiv$ $Q^{L}(\mathbf{e}) \cup Q^{J F}(\mathbf{e}) \cup Q^{P A}(\mathbf{e})$ for each $\mathbf{e} \in \mathcal{E}$. According to Proposition A1 in Appendix 1, there exists an ESOF which satisfies the axioms LS, J-RF, and PA if and only if $Q^{L J P}(\mathbf{e})$ is consistent for each $\mathbf{e} \in \mathcal{E} .^{9}$ Unfortunately, $Q^{L J P}(\mathbf{e})$ is not consistent for some $\mathbf{e} \in \mathcal{E}$. This is due to the following property:

Proposition 1: The union of any two of the relations $Q^{L}(\mathbf{e}), Q^{J F}(\mathbf{e})$, and $Q^{P A}(\mathbf{e})$ is inconsistent for some $\mathbf{e} \in \mathcal{E}$ and for any $J \in \mathcal{J}^{M E}$.

To begin with, the inconsistency of $Q^{L}(\mathbf{e}) \cup Q^{J F}(\mathbf{e})$ is easily confirmed by the fact that $Q^{L}(\mathbf{e})$ is interested solely in the wellness of allocation rules, whereas $Q^{J F}(\mathbf{e})$ represents the criterion which judges the wellness of extended alternatives, completely ignoring the wellness of allocation rules. A similar argument can be applied to the case of $Q^{L}(\mathbf{e}) \cup Q^{P A}(\mathbf{e})$.

How about the binary relation $Q^{J F}(\mathbf{e}) \cup Q^{P A}(\mathbf{e})$ ? This is related to the issue known as the problem of compatibility between fairness and efficiency in resource allocations, and its answer seems to depend on the criteria of distributive justice $J$. However, as the following example shows, if $J \in \mathcal{J}^{M E}$, then $Q^{J F}(\mathbf{e})$ and $Q^{P A}(\mathbf{e})$ are incompatible, regardless of what type of distributive justice this $J$ represents. ${ }^{10}$

Example 1: Let $N=\{1,2\}$ and $\bar{x}=3$. The production function is given by $f(x)=x$ for all $x \in \mathbb{R}_{+}$. Define an economic environment $\mathbf{e}=(\mathbf{u}, \mathbf{s}) \in \mathcal{E}$ as follows: Let $s_{i}=1$ for any $i \in N$. Consider the following four feasible allocations: $\mathbf{z}^{*}=((1,1),(1,1)), \mathbf{z}^{* *}=((2,2),(2,2)), \mathbf{z}^{*}(\theta)=((1,1+\theta),(1,1-\theta))$ , and $\mathbf{z}^{* *}(\theta)=((2,2-\theta),(2,2+\theta))$, where $\theta \in(0,1)$. The utility function of

\footnotetext{
${ }^{9}$ The definition of consistent binary relations is given by Definition 4 in Appendix 1 below.

${ }^{10}$ A similar incompatibility result is also obtained by Fleurbaey and Trannoy (2003).
} 
the individual 1 is assumed to have the following property: for $z=(x, y) \in Z$, if $z=z_{1}^{*}(\theta)$ or $z=z_{1}^{* *}$, then

$$
u_{1}(z)=(1-\theta+\varepsilon) \cdot(\bar{x}-x)+y, \text { where } \varepsilon>0 \text { is small enough; }
$$

and if $z=z_{1}^{* *}(\theta)$ or $z=z_{1}^{*}$, then

$$
u_{1}(z)=(1-\theta-\varepsilon) \cdot(\bar{x}-x)+y .
$$

Also, the utility function of the individual 2 is assumed to have the following property: for $z=(x, y) \in Z$ with $x \in[0,1)$,

$$
u_{2}(z)=(1-\theta) \cdot(\bar{x}-x)+y ;
$$

for $z=(x, y) \in Z$ with $x \in[1, \bar{x}]$, if $z=z_{2}^{* *}(\theta)$ or $z=z_{2}^{*}$, then

$$
u_{2}(z)=(1+\theta-\varepsilon) \cdot(\bar{x}-x)+y ;
$$

and if $z=z_{2}^{*}(\theta)$ or $z=z_{2}^{* *}$, then

$$
u_{2}(z)=(1+\theta+\varepsilon) \cdot(\bar{x}-x)+y .
$$

Figure 1 illustrates such a situation.

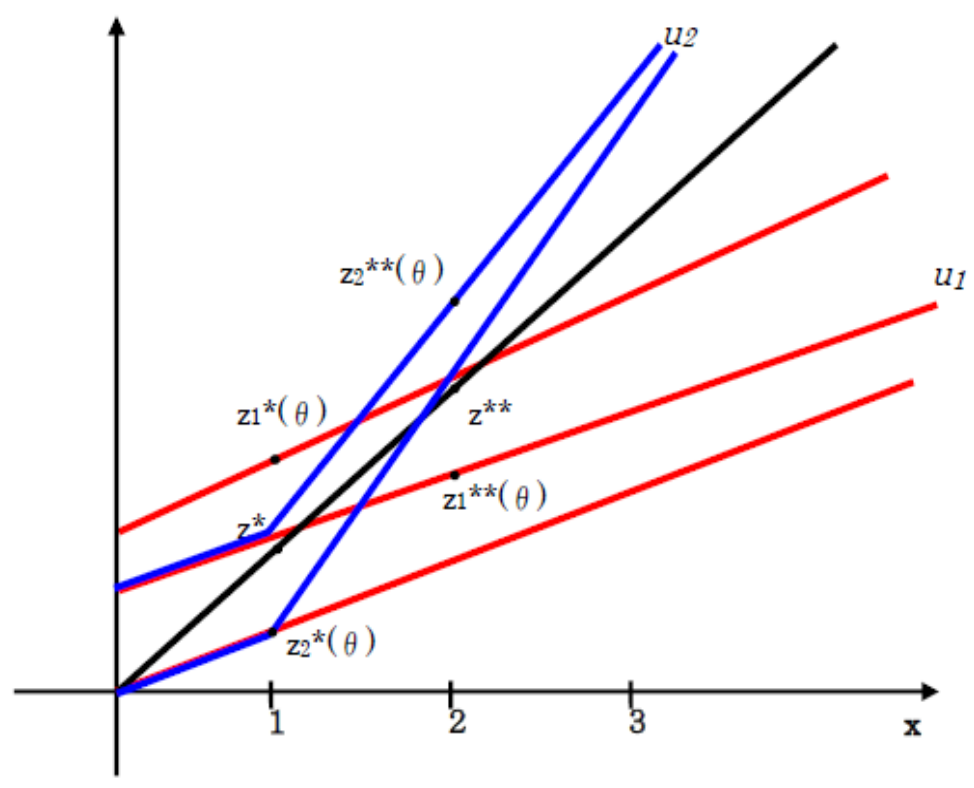

Example 1 in the consumption space

Figure 1 
Let $\gamma^{*}, \gamma^{* *}, \gamma^{*}(\theta)$, and $\gamma^{* *}(\theta)$ be the allocation rules respectively in which $\mathbf{z}^{*}, \mathbf{z}^{* *}, \mathbf{z}^{*}(\theta)$, and $\mathbf{z}^{* *}(\theta)$ become respectively Nash equilibrium outcomes under $\mathbf{e} \in \mathcal{E}$. Then, if $J \in \mathcal{J}^{M E}$, its corresponding $Q^{J F}(\mathbf{e})$ should have:

$\left(\left(\mathbf{z}^{*}, \gamma^{*}\right),\left(\mathbf{z}^{*}(\theta), \gamma^{*}(\theta)\right)\right) \in P\left(Q^{J F}(\mathbf{e})\right),\left(\left(\mathbf{z}^{* *}, \gamma^{* *}\right),\left(\mathbf{z}^{* *}(\theta), \gamma^{* *}(\theta)\right)\right) \in P\left(Q^{J F}(\mathbf{e})\right)$.

On the other hand, by the definition of $Q^{P A}(\mathbf{e})$, we have:

$\left(\left(\mathbf{z}^{*}(\theta), \gamma^{*}(\theta)\right),\left(\mathbf{z}^{* *}, \gamma^{* *}\right)\right) \in P\left(Q^{P A}(\mathbf{e})\right),\left(\left(\mathbf{z}^{* *}(\theta), \gamma^{* *}(\theta)\right),\left(\mathbf{z}^{*}, \gamma^{*}\right)\right) \in P\left(Q^{P A}(\mathbf{e})\right)$.

Thus, the binary relation $Q^{J F}(\mathbf{e}) \cup Q^{P A}(\mathbf{e})$ is not consistent.

Thus, this incompatibility can be applied for any $J$ representing any meaningful equity criterion, such as the 'equity as no-envy' ${ }^{11}$, and Sen's theory of "equality of capability. ${ }^{12}$ " This is because any meaningful equity criterion should meet ME.

Note as the above example shows, the incompatibility between $Q^{J F}(\mathbf{e})$ and $Q^{P A}(\mathbf{e})$ is obtained by using the weak Pareto principle only and without any help of the Pareto indifference condition. The Pareto indifference condition is not a crucial factor for this incompatibility.

\section{On Possibility of Second-Best Extended Social Ordering Functions}

So far, the previous section showed that there is no ESOF which satisfies the three basic axioms, LS, J-RF, PA, simultaneously. Then, the next step is to examine the possibility of the second-best ESOFs which satisfy some weaker requirements of the three basic axioms. There are at least two types of methods used to solve this problem. The first method is based on the pluralistic application of axioms proposed by Sen and Williams (1982). The second method is based on the lexicographic application of axioms. The formal definitions of these approaches are given in Appendix 2.

Here, we focus on the lexicographic application, which sometimes appeared in the literature of normative theories such as Rawls (1971) and van Parijs (1995). The lexicographic application takes one priority order among axioms, and then makes a ranking between any two alternatives in accordance with the first prior axiom. Then, if the pair of alternatives is non-comparable with respect to the first prior axiom, then the second prior axiom is applied for ranking them. In the following discussion, we will show that even according to this lexicographic application, we cannot yet generally construct a consistent

${ }^{11}$ The example of the latest successful research is Tadenuma (2002). Also, see Yoshihara (2005).

${ }^{12}$ In fact, Gotoh, Suzumura, and Yoshihara (2005) show that if $J$ represents Sen's theory of "equality of capability," then J-RF is incompatible with PA. 
ESOFs. Then, we will consider a further concession to construct the secondbest ESOFs. It is a weaker variant of lexicographic application in the sense that the second prior axiom is applied only to a subset, not to the whole set, of non-comparable pairs of the first prior axiom. Based on this method, we will show the existence of four types of the second-best ESOFs.

For any $\mathbf{e} \in \mathcal{E}$ and any binary relation $Q(\mathbf{e}) \subseteq\left(Z^{n} \times \Gamma\right)^{2}$, let $N(Q(\mathbf{e})) \subseteq$ $\left(Z^{n} \times \Gamma\right)^{2}$ be defined by: for any $(\mathbf{z}, \gamma),\left(\mathbf{z}^{\prime}, \gamma^{\prime}\right) \in \mathcal{R}(\mathbf{e})$,

$$
\left((\mathbf{z}, \gamma),\left(\mathbf{z}^{\prime}, \gamma^{\prime}\right)\right) \in N(Q(\mathbf{e})) \Leftrightarrow\left((\mathbf{z}, \gamma),\left(\mathbf{z}^{\prime}, \gamma^{\prime}\right)\right) \notin Q(\mathbf{e}) \&\left(\left(\mathbf{z}^{\prime}, \gamma^{\prime}\right),(\mathbf{z}, \gamma)\right) \notin Q(\mathbf{e}) .
$$

Note that this relation is the non-comparable part of $Q(\mathbf{e})$. To see the possibility of the second-best ESOFs based on the lexicographic application, let us first examine J-RF first-PA second priority rule, which is to consider a binary relation function $Q_{l e x}^{J \vdash P}: \mathcal{E} \rightarrow\left(Z^{n} \times \Gamma\right)^{2}$, defined as follows: for any $\mathbf{e} \in \mathcal{E}$

$$
\begin{aligned}
Q_{l e x}^{J \vdash P}(\mathbf{e}) & \equiv Q^{J F}(\mathbf{e}) \cup\left[N\left(Q^{J F}(\mathbf{e})\right) \cap Q^{P A}(\mathbf{e})\right] ; \text { and } \\
P\left(Q_{\text {lex }}^{J \vdash P}(\mathbf{e})\right) & \equiv P\left(Q^{J F}(\mathbf{e})\right) \cup\left[N\left(Q^{J F}(\mathbf{e})\right) \cap P\left(Q^{P A}(\mathbf{e})\right)\right] .
\end{aligned}
$$

The relation $Q_{\text {lex }}^{J \vdash P}$ (e) ranks any two extended alternatives by applying the axiom $\mathbf{J}-\mathbf{R F}$ in the first place, and if this pair belongs to the non-comparable part of $Q^{J F}(\mathbf{e})$, then PA is applied to rank them. In a similar way, we can also consider PA first-J-RF second priority rule, and define the binary relation function $Q_{l e x}^{P \vdash J}: \mathcal{E} \rightarrow\left(Z^{n} \times \Gamma\right)^{2}$.

Unfortunately, we still obtain the following impossibilities:

Proposition 2: Both $Q_{\text {lex }}^{J \vdash P}(\mathbf{e})$ and $Q_{\text {lex }}^{P \vdash J}(\mathbf{e})$ are inconsistent for some $\mathbf{e} \in \mathcal{E}$, if $J \in \mathcal{J}^{M E}$.

This is checked by using the same four feasible allocations and the same economic environment as in Example 1. In fact, we can see in Example 1 that

$\left(\left(\mathbf{z}^{*}, \gamma^{*}\right),\left(\mathbf{z}^{*}(\theta), \gamma^{*}(\theta)\right)\right) \in N\left(Q^{P A}(\mathbf{e})\right) \&\left(\left(\mathbf{z}^{* *}, \gamma^{* *}\right),\left(\mathbf{z}^{* *}(\theta), \gamma^{* *}(\theta)\right)\right) \in N\left(Q^{P A}(\mathbf{e})\right)$,

which implies that the discussion of inconsistency in Example $\mathbf{1}$ can be applied to $Q_{l e x}^{P \vdash J}(\mathbf{e})$. The same discussion is applied to the binary relation $Q_{\text {lex }}^{J \vdash P}(\mathbf{e})$.

As Proposition 2 indicates, we cannot construct any second-best ESWF based on the lexicographic application. To secure the existence of a compatible lexicographic combination of our basic axioms, a further concession seems to be required. As such one, let us consider, for each $\mathbf{e} \in \mathcal{E}$, to choose appropriately a subset $N^{*}\left(Q^{J F}(\mathbf{e})\right)$ from the whole set of non-comparable parts, $N\left(Q^{J F}(\mathbf{e})\right)$, in order to make $Q^{J F}(\mathbf{e}) \cup\left[N^{*}\left(Q^{J F}(\mathbf{e})\right) \cap Q^{P A}(\mathbf{e})\right]$ consistent. Given $J \in \mathcal{J}$ and $\mathbf{x} \in[0, \bar{x}]^{n}$, let 
$B(J(\mathbf{e}) ; \mathbf{x}) \equiv\left\{(\mathbf{x}, \mathbf{y}) \in Z(\mathbf{s}) \mid \forall\left(\mathbf{x}, \mathbf{y}^{\prime}\right) \in Z(\mathbf{s}):\left(\left(\mathbf{x}, \mathbf{y}^{\prime}\right),(\mathbf{x}, \mathbf{y})\right) \notin P(J(\mathbf{e}))\right\}$.

For any $\mathbf{e} \in \mathcal{E}$ and any $(\mathbf{z}, \gamma),\left(\mathbf{z}^{\prime}, \gamma^{\prime}\right) \in \mathcal{R}(\mathbf{e})$, let $N^{*}\left(Q^{J F}(\mathbf{e})\right) \subsetneq$ $N\left(Q^{J F}(\mathbf{e})\right)$ be given as follows:

$\left((\mathbf{z}, \gamma),\left(\mathbf{z}^{\prime}, \gamma^{\prime}\right)\right) \in N^{*}\left(Q^{J F}(\mathbf{e})\right) \Leftrightarrow \mathbf{x} \neq \mathbf{x}^{\prime} \& \mathbf{z} \in B(J(\mathbf{e}) ; \mathbf{x}) \& \mathbf{z}^{\prime} \in B\left(J(\mathbf{e}) ; \mathbf{x}^{\prime}\right)$.

That is, $\left((\mathbf{z}, \gamma),\left(\mathbf{z}^{\prime}, \gamma^{\prime}\right)\right)$ is a non-comparable pair in the sense of $N^{*}\left(Q^{J F}(\mathbf{e})\right)$ if and only if $\mathbf{z}$ and $\mathbf{z}^{\prime}$ of this pair have mutually different labor supplies, $\mathbf{x} \neq \mathbf{x}^{\prime}$, and each of them is a $J(\mathbf{e})$-maximal allocation within the same profile of labor supplies. Note that the condition $\mathbf{x} \neq \mathbf{x}^{\prime}$ implies that $N^{*}\left(Q^{J F}(\mathbf{e})\right)$ is a subset of $N\left(Q^{J F}(\mathbf{e})\right)$. Then, for any $\mathbf{e} \in \mathcal{E}$, let:

$$
\begin{aligned}
Q_{l e x}^{* J \vdash P}(\mathbf{e}) & \equiv Q^{J F}(\mathbf{e}) \cup\left[N^{*}\left(Q^{J F}(\mathbf{e})\right) \cap Q^{P A}(\mathbf{e})\right] ; \\
P\left(Q_{l e x}^{* J \vdash P}(\mathbf{e})\right) & \equiv P\left(Q^{J F}(\mathbf{e})\right) \cup\left[N^{*}\left(Q^{J F}(\mathbf{e})\right) \cap P\left(Q^{P A}(\mathbf{e})\right)\right] .
\end{aligned}
$$

The relation $Q_{l e x}^{* J \vdash P}(\mathbf{e})$ ranks any two extended alternatives by applying the axiom J-RF in the first place, and if this pair belongs to the specific noncomparable part, $N^{*}\left(Q^{J F}(\mathbf{e})\right)$, then $\mathbf{P A}$ is applied to rank them.

Next, let us define an appropriate subset, $N^{*}\left(Q^{P A}(\mathbf{e})\right)$, of $N\left(Q^{P A}(\mathbf{e})\right)$ to make $Q^{P A}(\mathbf{e}) \cup\left[N^{*}\left(Q^{P A}(\mathbf{e})\right) \cap Q^{J F}(\mathbf{e})\right]$ consistent. For any $\mathbf{e} \in \mathcal{E}$, let $\partial S(\mathbf{e}) \equiv\left\{\overline{\mathbf{u}} \in \mathbb{R}^{n} \mid \exists \mathbf{z} \in P(\mathbf{e}): u_{i}\left(z_{i}\right)=\bar{u}_{i}(\forall i \in N)\right\}$. Then, for each $\overline{\mathbf{u}} \in$ $\partial S(\mathbf{e})$, let us select only one allocation $\mathbf{z}^{\overline{\mathbf{u}}} \in P(\mathbf{e})$ such that for each $i \in$ $N, u_{i}\left(z_{i}^{\overline{\mathbf{u}}}\right)=\bar{u}_{i}$. Now, let $P^{s}(\mathbf{e}) \equiv\left\{\mathbf{z}^{\overline{\mathbf{u}}}\right\}_{\overline{\mathbf{u}} \in \partial S(\mathbf{e})}$. By definition, $P^{s}(\mathbf{e}) \subseteq$ $P(\mathbf{e})$. Note that for any $\mathbf{z}^{\overline{\mathbf{u}}}, \mathbf{z}^{\overline{\mathbf{u}}^{\prime}} \in P^{s}(\mathbf{e}), \overline{\mathbf{u}} \neq \overline{\mathbf{u}}^{\prime}$. Then, for any $\mathbf{e} \in \mathcal{E}$, let $N^{*}\left(Q^{P A}(\mathbf{e})\right) \subseteq N\left(Q^{P A}(\mathbf{e})\right)$ be such that

$$
\left((\mathbf{z}, \gamma),\left(\mathbf{z}^{\prime}, \gamma^{\prime}\right)\right) \in N^{*}\left(Q^{P A}(\mathbf{e})\right) \Leftrightarrow \mathbf{z}, \mathbf{z}^{\prime} \in P^{s}(\mathbf{e}) \text { and } \mathbf{z} \neq \mathbf{z}^{\prime} .
$$

That is, $\left((\mathbf{z}, \gamma),\left(\mathbf{z}^{\prime}, \gamma^{\prime}\right)\right)$ is a non-comparable pair in the sense of $N^{*}\left(Q^{P A}(\mathbf{e})\right)$ if and only if $\mathbf{z}$ and $\mathbf{z}^{\prime}$ of this pair are different Pareto efficient allocations, and moreover, their corresponding utility allocations are also different. Note that the latter property follows from $\mathbf{z}, \mathbf{z}^{\prime} \in P^{s}(\mathbf{e})$. By this property, $\mathbf{z}$ and $\mathbf{z}^{\prime}$ are Pareto non-comparable, so that $N^{*}\left(Q^{P A}(\mathbf{e})\right)$ is actually a subset of $N\left(Q^{P A}(\mathbf{e})\right)$. Then, for any $\mathbf{e} \in \mathcal{E}$, let:

$$
\begin{aligned}
Q_{l e x}^{* P \vdash J}(\mathbf{e}) & \equiv Q^{P A}(\mathbf{e}) \cup\left[N^{*}\left(Q^{P A}(\mathbf{e})\right) \cap Q^{J F}(\mathbf{e})\right] ; \\
P\left(Q_{l e x}^{* P \vdash J}(\mathbf{e})\right) & \equiv P\left(Q^{P A}(\mathbf{e})\right) \cup\left[N^{*}\left(Q^{P A}(\mathbf{e})\right) \cap P\left(Q^{J F}(\mathbf{e})\right)\right] .
\end{aligned}
$$

The relation $Q_{l e x}^{* P \vdash J}(\mathbf{e})$ ranks over any two extended alternatives by applying the axiom PA in the first place, and if this pair belongs to the specific noncomparable part, $N^{*}\left(Q^{P A}(\mathbf{e})\right)$, then $\mathbf{J}-\mathbf{R F}$ is applied to rank them.

Using these concessive lexicographic binary relation functions, we obtain: 
Theorem 1: Let $J \in \mathcal{J}^{M E}$, and for each $\mathbf{e} \in \mathcal{E}, J(\mathbf{e})$ be a continuous quasiordering on $Z(\mathbf{s})^{13}$ such that for each $\mathbf{x} \in[0, \bar{x}]^{n}, B(J(\mathbf{e}) ; \mathbf{x})$ is a singleton. Then, there exist at least four ESOFs such that each of which contains either of the following binary relation functions as subrelation mappings:
(i) $Q_{\text {lex }}^{L \vdash(* P \vdash J)}$;
(ii) $Q_{l e x}^{(* P \vdash J) \vdash L}$;
(iii) $Q_{l e x}^{L \vdash(* J \vdash P)}$; and (iv) $Q_{l e x}^{(* J \vdash P) \vdash L}$.

Let $Q^{L \vdash(* P \vdash J)}$ (resp. $Q^{L \vdash(* J \vdash P)}$ ) be an ESOF which is obtained as an ordering extension of $Q_{l e x}^{L \vdash(* P \vdash J)}$ (resp. $\left.Q_{l e x}^{L \vdash(* J \vdash P)}\right)$. Note that both $Q^{L \vdash(* P \vdash J)}$ and $Q^{L \vdash(* J \vdash P)}$ are interesting from the viewpoint of non-welfaristic normative theories. Both of them are given by the weaker sense of lexicographic application as discussed above, and give the first priority to a non-consequential axiom LS rather than the other two consequentialist axioms. Both the Rawlsian two principles of justice combined with the Pareto principle and the Real Libertarianism [van Parijs (1995)] combined with the Pareto principle would be formalized as the $Q^{L \vdash(* P \vdash J)}$-type or the $Q^{L \vdash(* J \vdash P)}$-type.

\subsection{Rationally Chosen Allocation Rules via ESOFs}

In this subsection, we characterize allocation rules rationally chosen via $Q^{L \vdash(* P \vdash J)}$ and/or $Q^{L \vdash(* J \vdash P)}$. Given any $Q \in \mathcal{Q}$, the rational choice set $C(Q)$ of allocation rules associated with $Q$ is defined by:

$\gamma \in C(Q) \Leftrightarrow \forall \mathbf{e} \in \mathcal{E}, \exists \mathbf{z} \in \tau(\gamma, \mathbf{e})$ s.t. $\forall\left(\mathbf{z}^{\prime}, \gamma^{\prime}\right) \in \mathcal{R}(\mathbf{e}),\left((\mathbf{z}, \gamma),\left(\mathbf{z}^{\prime}, \gamma^{\prime}\right)\right) \in Q(\mathbf{e})$.

What kinds of allocation rules can be rationally chosen via ESOFs $Q^{L \vdash(* P \vdash J)}$ and/or $Q^{L \vdash(* J \vdash P)}$ ? If $\gamma \in C\left(Q^{L \vdash(* P \vdash J)}\right) \cup C\left(Q^{L \vdash(* J \vdash P)}\right)$, then what kinds of feasible allocations can this $\gamma$ implement in Nash equilibria? To examine such questions, let a rationally chosen allocation rule $\gamma \in$ $C\left(Q^{L \vdash(* P \vdash J)}\right) \cup C\left(Q^{L \vdash(* J \vdash P)}\right)$ be called the first best allocation rule if for any $\mathbf{e} \in \mathcal{E}$ and any $\mathbf{z} \in \tau(\gamma, \mathbf{e}), \mathbf{z}$ is Pareto efficient and $\mathbf{z} \in B(J(\mathbf{e}) ; \mathbf{x})$. Our particular interest is the existence issue of the first best allocation rule rationalized by $Q^{L \vdash(* P \vdash J)}$ and/or $Q^{L \vdash(* J \vdash P)}$.

Let us call $\gamma=(M, g) \in \Gamma$ an efficient allocation rule if, for any $\mathbf{e} \in \mathcal{E}$, $\mathbf{z} \in \tau(\gamma, \mathbf{e})$ implies $\mathbf{z} \in P(\mathbf{e})$. Denote the subclass of $\Gamma$ which consists solely of efficient allocation rules by $\Gamma_{P E}$. Let us call $\gamma=(M, g) \in \Gamma$ a $J$-fair allocation rule if, for any $\mathbf{e} \in \mathcal{E}, \mathbf{z} \in \tau(\gamma, \mathbf{e})$ implies $\mathbf{z} \in B(J(\mathbf{e}) ; \mathbf{x})$. Denote the subclass of $\Gamma$ which consists solely of $J$-fair allocation rules by $\Gamma_{J F}$. Then:

Theorem 2: Let $J \in \mathcal{J}^{M O E}$, and for each $\mathbf{e} \in \mathcal{E}, J(\mathbf{e})$ be a continuous ordering on $Z(\mathbf{s})$ such that for each $\mathbf{x} \in[0, \bar{x}]^{n}, B(J(\mathbf{e}) ; \mathbf{x})$ is a singleton. Then, there exists an ESOF $Q^{L \vdash(* J \vdash P)}$ (resp. $\left.Q^{L \vdash(* P \vdash J)}\right)$ such that for each $\mathbf{e} \in \mathcal{E}, Q^{L \vdash(* J \vdash P)}(\mathbf{e}) \supseteq Q_{l e x}^{L \vdash(* J \vdash P)}(\mathbf{e})\left(\right.$ resp. $\left.Q^{L \vdash(* P \vdash J)}(\mathbf{e}) \supseteq Q_{\text {lex }}^{L \vdash(* P \vdash J)}(\mathbf{e})\right)$,

\footnotetext{
${ }^{13}$ A quasi-ordering $R$ is continuous on $X$ if for any $x \in X$, its upper and lower contour sets at $R$ is open.
} 
and $\varnothing \neq C\left(Q^{L \vdash(* J \vdash P)}\right)=\Gamma_{L} \cap \Gamma_{P E} \cap \Gamma_{J F} \quad\left(\right.$ resp. $\varnothing \neq C\left(Q^{L \vdash(* P \vdash J)}\right) \supseteq$ $\left.\Gamma_{L} \cap \Gamma_{P E} \cap \Gamma_{J F}\right)$.

Theorem 2 shows that if $J \in \mathcal{J}^{M O E}$, then the rationally chosen allocation rule via $Q^{L \vdash(* J \vdash P)}$ has the following desired properties: it is labor sovereign, and implements Pareto efficient and $J$-fair allocations in Nash equilibria. The same property holds for the case of $Q^{L \vdash(* P \vdash J)}$.

This characterization in Theorem 2 is due to the objective egalitarianism of $J$. If $J \in \mathcal{J}^{M O E}$, then $B(J(\mathbf{e}) ; \mathbf{x})=B\left(J\left(\mathbf{e}^{\prime}\right) ; \mathbf{x}\right)$ for any $\mathbf{x} \in[0, \bar{x}]^{n}$ and any $\mathbf{e}, \mathbf{e}^{\prime} \in \mathcal{E}$ with $\mathbf{s}=\mathbf{s}^{\prime}$. This invariance property of $B(J(\cdot) ; \mathbf{x})$ plays an important role in the existence issue of the first best allocation rules rationalized by $Q^{L \vdash(* J \vdash P)}$ and/or $Q^{L \vdash(* P \vdash J)}$. In contrast, if $J \in \mathcal{J}^{M E} \backslash \mathcal{J}^{M O E}$, then the existence of the first best allocation rules rationalized by $Q^{L \vdash(* J \vdash P)}$ and/or $Q^{L \vdash(* P \vdash J)}$ is not necessarily guaranteed. For instance, let $J^{U D} \in$ $\mathcal{J}^{M E} \backslash \mathcal{J}^{M O E}$ represent undominated diversity. Then, there is no first best allocation rule in terms of Pareto efficiency and $J^{U D}$-fairness, so that the corresponding rationally chosen allocation rule does not have such desired properties. ${ }^{14}$

\section{Discussion}

In this section, we provide some remarks on the relevant literature of ESOFs. As mentioned in the introduction, Kaplow and Shavell (2001) and Blackorby, Bossert, and Donaldson (2005) also discuss different types of 'extended' social ordering functions.

For instance, Kaplow and Shavell (2001) define any "non-welfarist" axiom as being incompatible with the Pareto Indifference principle. Then, they show that if a social welfare function satisfies continuity and such a "non-welfarist" axiom, then it violates the weak Pareto principle. This is derived from the fact that the continuity of the social welfare function and the weak Pareto principle immediately imply the Pareto Indifference principle. ${ }^{15}$ Blackorby et al. (2005) show that if a social welfare function defined over the domain of multi-profiles satisfies Universal Domain, Pareto Indifference, and Binary Independence of Irrelevant Alternatives, then it implies Strong Neutrality. Note that Strong Neutrality is regarded as the axiom of Welfarism.

It is well known that, even in the case of B-S social welfare functions with the domains of the utility profiles only, Sen (1977) and Roberts (1980) show that the conventional Arrovian axioms of universal domain, Pareto indifference, and binary independence of irrelevant alternatives together imply strong neutrality. The crucial difference of Blackorby et al. (2005) from Sen

\footnotetext{
$\overline{14}$ This point is discussed in Yoshihara (2006).

${ }^{15}$ Kaplow and Shavell (2001) consider the Pareto indifference principle as the definition of welfarism for social welfare functions.
} 
(1977) and Roberts (1980) is that the former defines Binary Independence of Irrelevant Alternatives as requiring the social ranking of any two alternatives to depend on not only the utility information but also the non-welfaristic information associated with those two alternatives only. Hence, the independence axiom of Blackorby et al. (2005) is non-welfarist in nature, and it is weaker than the Sen-Roberts independence axiom. Nevertheless, Blackorby et al. (2005) conclude that even in such a framework, the possible social ordering function is only welfarist in nature, if it is required to satisfy the other Arrovian axioms such as Universal Domain and Pareto Indifference. This seems to provides us with a strong justification of welfarism.

We review the relationship between our approach and the above mentioned works briefly. First, the welfarist theorem of Blackorby et al. (2005) relies strongly on the axiom of Universal Domain. Such a domain condition cannot directly be applied to the resource allocation problems this paper considers here. For instance, in this paper, all available utility functions are restricted so as to be strongly monotonic and quasi-concave. In fact, as shown in Yoshihara (2006a), if a reasonable domain restriction is imposed, then an ESOF of $Q^{L \vdash(* P \vdash J)}$-type exists and it satisfies the independence axiom of Blackorby et al. (2005). The domain of this ESOF is restricted, because 1) the domain of welfarist information $\mathcal{U}^{n}$ is restricted to the class of profiles of continuous, strictly monotonic, and quasi-concave utility functions, and 2) the domain of non-welfarist information is also restricted.

Thus, our result on the possibility of the non-welfarist ESOFs is compatible with the result of Blackorby et al. (2005). Moreover, I believe that the universal domain assumption of the non-welfaristic information is not sound from an ethical point of view. This is because a well-being indicator expressing non-welfaristic information should be defined as a binary relation function characterized by a system of axioms ${ }^{16}$ so it needs different formal treatment from the welfarist indicator (individual utility functions) representing capricious subjective preferences.

Secondly, the conclusion of Kaplow and Shavell (2001) gives us basically the same message as that of Example 1 in this paper. However, Example 1 does not suppose the continuity of social ordering, contrary to the assumption of Kaplow and Shavell (2001). It is also worth noting that this resulting impossibility does not imply a justification of welfarism at all. This is because, as Fleurbaey, Tungodden, and Chang (2003) point out, the Pareto indifference principle and the welfarist axiom are not equivalent. In fact, our ESOF $Q^{(* P \vdash J) \vdash L}$ in Theorem 1 satisfies the weak Pareto principle as well as the Pareto indifference principle, and it also has the properties of the two types of non-welfarism $(J-\mathbf{R F}$ and $\mathbf{L S})$. However, this type of function does not meet the continuity axiom. This implies that the real factor inducing the

\footnotetext{
${ }^{16}$ An example of this is a series of works by Pattanaik and Xu (1990), where the ranking over opportunity sets is characterized by the system of axioms which reflect the viewpoint of "freedom of choice."
} 
impossibility is not the trade-off between welfarism and non-welfarism, but rather the requirement of continuity.

To summarize this, despite the conclusions of Kaplow and Shavell (2001) and Blackorby et al. (2005), it is eminently possible to construct a desirable social ordering function that has the properties of the welfarist Pareto principle and the non-welfarist criteria.

\section{Conclusion}

In the previous sections, we discussed that the welfarist's framework developed in traditional welfare economics provided us with a rather limited perspective for social evaluation, so a more comprehensive framework would be necessary. As such, we proposed the extended framework within which not only welfarist consequential values, but also non-welfarist consequential values and non-consequential values can be taken into consideration. Moreover, we introduced extended social ordering functions and, as axioms of which, Labor Sovereignty, Respect for $\mathbf{J}$-based Fairness based on non-welfaristic well-being notions, and the Pareto principle. Then, we showed a method of applying these axioms based on a weaker lexicographic approach, by which some consistent extended social ordering functions can be constructed in order to be compatible with the above three values.

\section{Appendix 1}

In this Appendix 1, the elementary properties of binary relations are provided, which constitute an analytical technique useful to consider the existence issue of ESOFs. Let $X$ be the universal set of any alternatives and $R$ be a binary relation defined over this set. If $R$ satisfies completeness and transitivity in particular, we shall call it an ordering. Also:

Definition 2: An axiom a is represented by a binary relation $R^{a} \subseteq X \times X$ if the following condition holds: for any $\mathbf{x}, \mathbf{x}^{\prime} \in X$,

$\left(\mathbf{x}, \mathbf{x}^{\prime}\right) \in R^{a} \Leftrightarrow$ according to the axiom $a, \mathbf{x}$ is at least desired as $\mathbf{x}^{\prime}$;

$\left(\mathbf{x}, \mathbf{x}^{\prime}\right) \in P\left(R^{a}\right) \Leftrightarrow$ according to the axiom $a, \mathbf{x}$ is strictly desirable than $\mathbf{x}^{\prime}$.

In general, the binary relation representing an axiom is not necessarily a complete ordering. In the following discussion, let us denote the representation of the axiom $a$ by $R^{a}$. Then, let us see how an ordering $R \subseteq X \times X$ satisfies an axiom in general.

Definition 3: $A$ binary relation $R$ satisfies a class of axioms $\left\{a^{\lambda}\right\}_{\lambda \in \Lambda}$ if the following condition holds: 


$$
R \supseteq\left[\cup_{\lambda \in \Lambda} R^{a^{\lambda}}\right] \text { and } P(R) \supseteq\left[\cup_{\lambda \in \Lambda} P\left(R^{a^{\lambda}}\right)\right] \text {. }
$$

As Definition 3 suggests, a binary relation $R$ satisfies axioms $a^{1}, \ldots, a^{m}$ if and only if it contains all of the axiom-representing relations $R^{a^{1}}, \ldots, R^{a^{m}}$ as its subrelations.

Given a class of axioms on ordering relations, one interesting problem is to examine whether there exists an ordering relation that satisfies all of these axioms. To discuss this question, the following notion is crucial:

Definition 4 [Suzumura (1976)]: A binary relation $R \subseteq X \times X$ is consistent if , for any finite subset $\left\{x^{1}, x^{2}, \ldots, x^{t}\right\}$ of $X$, the following condition does not hold:

$$
\left[\left(x^{1}, x^{2}\right) \in P(R),\left(x^{k}, x^{k+1}\right) \in R(\forall k=2, \ldots, t-1)\right] \Rightarrow\left(x^{t}, x^{1}\right) \in R .
$$

Proposition A1: There exists an ordering relation $R \subseteq X \times X$ which satisfies a class of axioms $\left\{a^{\lambda}\right\}_{\lambda \in \Lambda}$ if and only if $\left[\cup_{\lambda \in \Lambda} R^{a^{\lambda}}\right]$ is consistent.

According to Proposition A1, it is sufficient to confirm whether or not the union of the axiom-representing relations $\left\{R^{a^{\lambda}}\right\}_{\lambda \in \Lambda}$ meets the consistency. This condition can be useful when we discuss the existence of ESOFs satisfying some classes of axioms.

\section{Appendix 2}

1. The pluralistic application of axioms [Sen and Williams (1982)]

Given any two axioms $a$ and $b$ which are mutually incompatible, the pluralistic application of axioms is to construct a binary relation $R^{a \cap b} \subseteq X \times X$ which is defined as: $R^{a \cap b} \equiv R^{a} \cap R^{b}$ and $P\left(R^{a \cap b}\right) \equiv\left[P\left(R^{a}\right) \cap R^{b}\right] \cup$ $\left[R^{a} \cap P\left(R^{b}\right)\right]$. Then, $R^{a \cap b}$ becomes consistent whenever $R^{a}$ and $R^{b}$ are respectively consistent. Thus, this kind of second best resolution is to consider an ordering extension of $R^{a \cap b}$.

2. The lexicographic application of axioms

Given any binary relation $R$, let $N(R) \subseteq X \times X$ be defined as follows: for any $\mathbf{x}, \mathbf{x}^{\prime} \in X,\left(\mathbf{x}, \mathbf{x}^{\prime}\right) \in N(R) \Leftrightarrow\left(\mathbf{x}, \mathbf{x}^{\prime}\right) \notin R$ and $\left(\mathbf{x}^{\prime}, \mathbf{x}\right) \notin R .{ }^{17}$ Given any two axioms $a$ and $b$ which are mutually incompatible, the lexicographic application of axioms is to construct a binary relation $R_{\text {lex }}^{a \vdash b} \subseteq X \times X$ which is defined by: for any $x, x^{\prime} \in X$,

$$
\begin{aligned}
& \left(x, x^{\prime}\right) \in R_{\text {lex }}^{a \vdash b} \Leftrightarrow\left(x, x^{\prime}\right) \in R^{a} \cup\left[N\left(R^{a}\right) \cap R^{b}\right] ; \text { and } \\
& \left(x, x^{\prime}\right) \in P\left(R_{\text {lex }}^{a \vdash b}\right) \Leftrightarrow\left(x, x^{\prime}\right) \in P\left(R^{a}\right) \cup\left[N\left(R^{a}\right) \cap P\left(R^{b}\right)\right] .
\end{aligned}
$$

$\overline{17}$ The definition of this binary relation is based on Suzumura (2004). 
That is, suppose that the society gives a priority to axiom $a$ rather than to axiom $b$. Then, for any two alternatives, $a$ is applied by $R_{l e x}^{a \vdash b}$ in the first place to make a comparison between them, and $b$ is applied only if these two alternatives are incomparable by $a$. This is called axiom a first-axiom $b$ second priority rule.

According to Proposition A1, an ordering extension of $R_{\text {lex }}^{a \vdash b}$ is possible whenever $R_{l e x}^{a \vdash b}$ is consistent. Unfortunately, however, the consistency of $R_{l e x}^{a \vdash b}$ is not guaranteed in general. Thus, we need an algorithm to see what properties of the axioms $a$ and/or $b$ can make $R_{l e x}^{a \vdash b}$ consistent.

Suppose that $R_{\text {lex }}^{a \vdash b}$ is not consistent. Our strategy is to choose an appropriate subset $N^{*}\left(R^{a}\right)$ from $N\left(R^{a}\right)$ such that

$$
R_{l e x}^{* a \vdash b} \equiv R^{a} \cup\left[N^{*}\left(R^{a}\right) \cap R^{b}\right]
$$

becomes consistent. Then, the problem is to identify what conditions this $N^{*}\left(R^{a}\right)$ should satisfy so as to make $R_{\text {lex }}^{* a \vdash b}$ consistent. A general solution to this problem is given by Yoshihara (2005), and here we introduce a corollary of this solution given in Yoshihara (2005).

Definition 5 [Yoshihara (2005)]: Given a binary relation $R \subseteq X \times X$, a subset $N^{*}(R) \subseteq N(R)$ is said to be connected if for any $\left(x, x^{\prime}\right),\left(y, y^{\prime}\right) \in N^{*}(R)$, there exists $\left\{z^{1}, \ldots, z^{t}\right\} \subseteq X$ such that $z^{1}=x^{\prime}$, $z^{t}=y$, and $\left(z^{k}, z^{k+1}\right) \in$ $N^{*}(R)$ holds for any $k=1, \ldots, t-1$.

Proposition A2 [Yoshihara (2005)]: Let $R^{a}$ be a quasi-ordering over $X$. Then, if the relation $N^{*}\left(R^{a}\right) \subseteq N\left(R^{a}\right)$ is transitive and connected, then the relation $R_{\text {lex }}^{* a \vdash b} \subseteq X \times X$ is consistent for any quasi-ordering $R^{b} \subseteq X \times X$.

\section{Appendix 3: Proofs of Theorems 1 and 2}

Proof of Theorem 1: We can see that for any e $\in \mathcal{E}$, both $N^{*}\left(Q^{J F}(\mathbf{e})\right)$ and $N^{*}\left(Q^{P A}(\mathbf{e})\right)$ are connected and transitive, where the definition of connectedness is given in Definition 5 of Appendix 2. Hence, by Proposition A2 of Appendix 2, both $Q_{l e x}^{* J \vdash P}$ and $Q_{l e x}^{* P \vdash J}$ are consistent binary relation functions.

Lemma 1: For each $\mathbf{e} \in \mathcal{E}, \underset{\mathbf{x} \in[0, \bar{x}]^{n}}{\cup} B(J(\mathbf{e}) ; \mathbf{x})$ has a closed graph in $Z(\mathbf{s})$.

Proof. It can be shown in a similar way to Lemma 4 in Gotoh, Suzumura, and Yoshihara (2005).

Lemma 2 [Yoshihara (2000)]: For each $\mathbf{s} \in \mathcal{S}^{n}$, let $h:[0, \bar{x}]^{n} \rightarrow \mathbb{R}_{+}^{n}$ be a continuous function such that, for each $\mathbf{x} \in[0, \bar{x}]^{n}, h(\mathbf{x})=\mathbf{y}$ and $f\left(\sum s_{i} x_{i}\right)=\sum y_{i}$. Then, for any $\mathbf{e}=(\mathbf{u}, \mathbf{s}) \in \mathcal{E}$, there exists $\mathbf{x}^{*} \in[0, \bar{x}]^{n}$ such that $\left(\mathbf{x}^{*}, h\left(\mathbf{x}^{*}\right)\right)$ is a Pareto efficient allocation for $\mathbf{e}$. 
Proof. See Proposition 3 in Gotoh, Suzumura, and Yoshihara (2005).

Lemma 3: For each $\mathbf{e}=(\mathbf{u}, \mathbf{s}) \in \mathcal{E}$, there exists a Pareto efficient allocation $\mathbf{z}^{*} \in Z(\mathbf{s})$ such that $\mathbf{z}^{*} \in \underset{\mathbf{x} \in[0, \bar{x}]^{n}}{\bigcup} B(J(\mathbf{e}) ; \mathbf{x})$.

Proof. See Lemma 5 in Gotoh, Suzumura, and Yoshihara (2005).

Lemma 4 [Yoshihara (2000)]: Given $J \in \mathcal{J}^{M O E}$, for each e $\in \mathcal{E}$, let $P B^{J}(\mathbf{e}) \equiv P(\mathbf{e}) \cap\left[\bigcup_{\mathbf{x} \in[0, \bar{x}]^{n}} B(J(\mathbf{e}) ; \mathbf{x})\right]$. Let $h:[0, \bar{x}]^{n} \rightarrow \mathbb{R}_{+}^{n}$ be a continuous function such that, for each $\mathbf{x} \in[0, \bar{x}]^{n}, h(\mathbf{x})=\mathbf{y}$ and $f\left(\sum s_{i} x_{i}\right)=\sum y_{i}$. Then, there exists a game form $\gamma \in \Gamma_{L}$ such that, for any $\mathbf{e} \in \mathcal{E}, \mathbf{z} \in \tau(\gamma, \mathbf{e})$ holds if and only if $\mathbf{z}=(\mathbf{x}, h(\mathbf{x}))$, and it is Pareto efficient.

Proof. See Proposition 4 in Gotoh, Suzumura, and Yoshihara (2005).

Lemma 5: There exists $\gamma^{*} \in \Gamma_{L}$ such that $\tau\left(\gamma^{*}, \mathbf{e}\right)=P B^{J}(\mathbf{e})$ for all $\mathbf{e} \in \mathcal{E}$.

Proof. See the proof of Theorem 1 in Gotoh, Suzumura, and Yoshihara $(2005) .{ }^{18}$

Proof of Theorem 2: Given $\mathbf{e} \in \mathcal{E}$, let $S(\mathbf{e})$ be the utility possibility set of feasible allocations, and $\partial S(\mathbf{e})$ be its boundary. Since every utility function is strictly increasing, $\partial S(\mathbf{e})$ is the set of Pareto efficient utility allocations.

(1) Consider the case of $Q^{L \vdash(* J \vdash P)}$. Define an ordering $V(\mathbf{e})$ over $S(\mathbf{e})$ as follows:

1) if $\overline{\mathbf{u}}, \overline{\mathbf{u}}^{\prime} \in \partial S(\mathbf{e})$, then $\left(\overline{\mathbf{u}}, \overline{\mathbf{u}}^{\prime}\right) \in I(V(\mathbf{e}))$,

2) for any $\overline{\mathbf{u}}, \overline{\mathbf{u}}^{\prime} \in S(\mathbf{e})$, there exist $\mu, \mu^{\prime} \in[1,+\infty)$ such that $\mu \cdot \overline{\mathbf{u}}, \mu^{\prime} \cdot \overline{\mathbf{u}}^{\prime} \in \partial S(\mathbf{e})$ and $\left(\overline{\mathbf{u}}, \overline{\mathbf{u}}^{\prime}\right) \in V(\mathbf{e})$ if and only if $\mu \leq \mu^{\prime}$. This ordering $V(\mathbf{e})$ is continuous over $S(\mathbf{e})$.

Define a complete ordering $R_{\mathbf{e}, J}$ over $\underset{\mathbf{x} \in[0, \bar{x}]^{n}}{\cup} B(J(\mathbf{e}) ; \mathbf{x})$ as follows: for any $\mathbf{z}, \mathbf{z}^{\prime} \in \underset{\mathbf{x} \in[0, \bar{x}]^{n}}{\cup} B(J(\mathbf{e}) ; \mathbf{x}),\left(\mathbf{z}, \mathbf{z}^{\prime}\right) \in R_{\mathbf{e}, J} \Leftrightarrow\left(\mathbf{u}(\mathbf{z}), \mathbf{u}\left(\mathbf{z}^{\prime}\right)\right) \in V(\mathbf{u})$. This ordering $R_{\mathbf{e}, J}$ is continuous on $\underset{\mathbf{x} \in[0, \bar{x}]^{n}}{\cup} B(J(\mathbf{e}) ; \mathbf{x})$, and its maximal elements constitute $P B^{J}(\mathbf{e})$. Given $J \in \mathcal{J}^{M O E}$, let $J(\mathbf{e} ; \mathbf{x})$ be the restriction of $J(\mathbf{e})$ into the set of feasible allocations with $\mathbf{x}$.

Consider a binary relation $R_{\mathbf{e}, J} \cup\left[\bigcup_{\mathbf{x} \in[0, \bar{x}]^{n}} J(\mathbf{e} ; \mathbf{x})\right]$ over $Z(\mathbf{s})$. It is easy to see that this binary relation is consistent, so that there exists an ordering extension $R_{\mathbf{e}, J}^{*}$ of $R_{\mathbf{e}, J} \cup\left[\bigcup_{\mathbf{x} \in[0, \bar{x}]^{n}} J(\mathbf{e} ; \mathbf{x})\right]$ by Suzumura's (1976) extension theorem. Based upon this $R_{\mathbf{e}, J}^{*}$, let us consider an ordering function $Q^{L \vdash(* P \vdash J)}$ as follows: for each $\mathbf{e} \in \mathcal{E}$ and any $(\mathbf{z}, \gamma),\left(\mathbf{z}^{\prime}, \gamma^{\prime}\right) \in \mathcal{R}(\mathbf{e})$,

1) if $\gamma \in \Gamma_{L}$ and $\gamma^{\prime} \in \Gamma \backslash \Gamma_{L}$, then $\left((\mathbf{z}, \gamma),\left(\mathbf{z}^{\prime}, \gamma^{\prime}\right)\right) \in P\left(Q^{L \vdash(* J \vdash P)}(\mathbf{e})\right)$;

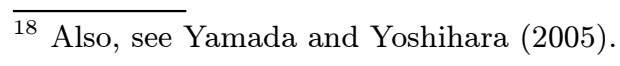


2) if either $\gamma, \gamma^{\prime} \in \Gamma_{L}$ or $\gamma, \gamma^{\prime} \in \Gamma \backslash \Gamma_{L}$, then

$$
\begin{aligned}
& \left((\mathbf{z}, \gamma),\left(\mathbf{z}^{\prime}, \gamma^{\prime}\right)\right) \in Q^{L \vdash(* J \vdash P)}(\mathbf{e}) \Leftrightarrow\left(\mathbf{z}, \mathbf{z}^{\prime}\right) \in R_{\mathbf{e}, J}^{*}, \\
& \left((\mathbf{z}, \gamma),\left(\mathbf{z}^{\prime}, \gamma^{\prime}\right)\right) \in P\left(Q^{L \vdash(* J \vdash P)}(\mathbf{e})\right) \Leftrightarrow\left(\mathbf{z}, \mathbf{z}^{\prime}\right) \in P\left(R_{\mathbf{e}, J}^{*}\right) .
\end{aligned}
$$

Note that $Q^{L \vdash(* J \vdash P)}(\mathbf{e})$ is complete and transitive, and $Q^{L \vdash(* J \vdash P)}(\mathbf{e}) \supseteq$ $Q_{\text {lex }}^{L \vdash(* J \vdash P)}(\mathbf{e})$ for each $\mathbf{e} \in \mathcal{E}$, by the definition. Finally, we can see that $C\left(Q^{L \vdash(* J \vdash P)}\right)=\Gamma_{L} \cap \Gamma_{P E} \cap \Gamma_{J F} \ni \gamma^{*}$.

(2) Consider the case of $Q^{L \vdash(* P \vdash J)}$. For each $\overline{\mathbf{u}} \in \partial S$ (e), let us select only one allocation $\mathbf{z}^{\overline{\mathbf{u}}} \in P(\mathbf{e})$ such that for each $i \in N, u_{i}\left(z_{i}^{\overline{\mathbf{u}}}\right)=\bar{u}_{i}$, and if for this $\overline{\mathbf{u}} \in$ $\partial S(\mathbf{e})$, there exists $\mathbf{z} \in P B^{J}(\mathbf{e})$ such that for each $i \in N, u_{i}\left(z_{i}\right)=\bar{u}_{i}$, then choose such an allocation as $\mathbf{z}^{\overline{\mathbf{u}}}$. Now, let $P^{s}(\mathbf{e}) \equiv\left\{\mathbf{z}^{\overline{\mathbf{u}}}\right\}_{\overline{\mathbf{u}} \in \partial S(\mathbf{e})}$. By definition, $P^{s}(\mathbf{e}) \subseteq P(\mathbf{e})$ and $P^{s}(\mathbf{e}) \cap P B^{J}(\mathbf{e}) \neq \varnothing$. Note that for any $\mathbf{z}^{\overline{\mathbf{u}}}, \mathbf{z}^{\overline{\mathbf{u}}^{\prime}} \in P^{s}(\mathbf{e})$, $\overline{\mathbf{u}} \neq \overline{\mathbf{u}}^{\prime}$.

Define an ordering $R_{\mathbf{e}, J}^{0}$ over $Z(\mathbf{s})$ as follows: for any $\mathbf{z}, \mathbf{z}^{\prime} \in Z(\mathbf{s})$,

(i) $\left(\mathbf{z}, \mathbf{z}^{\prime}\right) \in I\left(R_{\mathbf{e}, J}^{0}\right)$ if $\mathbf{z}, \mathbf{z}^{\prime} \in P^{s}(\mathbf{e}) \cap P B^{J}(\mathbf{e})$;

(ii) $\left(\mathbf{z}, \mathbf{z}^{\prime}\right) \in P\left(R_{\mathbf{e}, J}^{0}\right)$ if $\mathbf{z} \in P^{s}(\mathbf{e}) \cap P B^{J}(\mathbf{e})$ and $\mathbf{z}^{\prime} \in P^{s}(\mathbf{e}) \backslash P B^{J}(\mathbf{e})$;

(iii) $\left(\mathbf{z}, \mathbf{z}^{\prime}\right) \in R_{\mathbf{e}, J}^{0} \Leftrightarrow\left(\mathbf{z}, \mathbf{z}^{\prime}\right) \in J$ (e) for $\mathbf{z}, \mathbf{z}^{\prime} \in P^{s}(\mathbf{e}) \backslash P B^{J}(\mathbf{e})$;

(iv) $\left(\mathbf{z}, \mathbf{z}^{\prime}\right) \in P\left(R_{\mathbf{e}, J}^{0}\right)$ if there exist $\mu, \mu^{\prime} \in[1,+\infty)$ such that $\mu \cdot \mathbf{u}(\mathbf{z}), \mu^{\prime}$. $\mathbf{u}\left(\mathbf{z}^{\prime}\right) \in \partial S(\mathbf{u})$ and $\mu<\mu^{\prime}$; and

(v) $\left(\mathbf{z}, \mathbf{z}^{\prime}\right) \in R_{\mathbf{e}, J}^{0}$ if there exist $\mu, \mu^{\prime} \in[1,+\infty)$ such that $\mu \cdot \mathbf{u}(\mathbf{z}), \mu^{\prime} \cdot \mathbf{u}\left(\mathbf{z}^{\prime}\right) \in$ $\partial S(\mathbf{u})$ and $\mu=\mu^{\prime}$, and $\left(\mathbf{z}^{\mu \cdot \mathbf{u}(\mathbf{z})}, \mathbf{z}^{\mu^{\prime} \cdot \mathbf{u}\left(\mathbf{z}^{\prime}\right)}\right) \in R_{\mathbf{e}, J}$ for $\mathbf{z}^{\mu \cdot \mathbf{u}(\mathbf{z})}, \mathbf{z}^{\mu^{\prime} \cdot \mathbf{u}\left(\mathbf{z}^{\prime}\right)} \in$ $P^{s}(\mathbf{e})$.

Denote the set of maximal elements over $Z(\mathbf{s})$ in terms of $R_{\mathbf{e}, J}^{0}$ by $B\left(R_{\mathbf{e}, J}^{0}\right) \subseteq$ $Z(\mathbf{s})$. By definition, $P B^{J}(\mathbf{e}) \subseteq B\left(R_{\mathbf{e}, J}^{0}\right)$.

Based upon this $R_{\mathbf{e}, J}^{0}$, let us consider an ordering function $Q^{L \vdash(* P \vdash J)}$ as follows: for each $\mathbf{e} \in \mathcal{E}$ and any $(\mathbf{z}, \gamma),\left(\mathbf{z}^{\prime}, \gamma^{\prime}\right) \in \mathcal{R}(\mathbf{e})$,

1) if $\gamma \in \Gamma_{L}$ and $\gamma^{\prime} \in \Gamma \backslash \Gamma_{L}$, then $\left((\mathbf{z}, \gamma),\left(\mathbf{z}^{\prime}, \gamma^{\prime}\right)\right) \in P\left(Q^{L \vdash(* P \vdash J)}(\mathbf{e})\right)$;

2) if either $\gamma, \gamma^{\prime} \in \Gamma_{L}$ or $\gamma, \gamma^{\prime} \in \Gamma \backslash \Gamma_{L}$, then

$$
\begin{aligned}
& \left((\mathbf{z}, \gamma),\left(\mathbf{z}^{\prime}, \gamma^{\prime}\right)\right) \in Q^{L \vdash(* P \vdash J)}(\mathbf{e}) \Leftrightarrow\left(\mathbf{z}, \mathbf{z}^{\prime}\right) \in R_{\mathbf{e}, J}^{0}, \\
& \left((\mathbf{z}, \gamma),\left(\mathbf{z}^{\prime}, \gamma^{\prime}\right)\right) \in P\left(Q^{L \vdash(* P \vdash J)}(\mathbf{e})\right) \Leftrightarrow\left(\mathbf{z}, \mathbf{z}^{\prime}\right) \in P\left(R_{\mathbf{e}, J}^{0}\right) .
\end{aligned}
$$

Note that $Q^{L \vdash(* P \vdash J)}(\mathbf{e})$ is complete and transitive, and $Q^{L \vdash(* P \vdash J)}(\mathbf{e}) \supseteq$ $Q_{\text {lex }}^{L \vdash P \vdash J)}(\mathbf{e})$ for each $\mathbf{e} \in \mathcal{E}$, by the definition. Finally, we can see that $C\left(Q^{L \vdash(* P \vdash J)}\right) \supseteq \Gamma_{L} \cap \Gamma_{P E} \cap \Gamma_{J F} \ni \gamma^{*}$.

\section{References}

Arrow, K. J. (1963): Social Choice and Individual Values, 2nd ed., New York: Wiley. 
Blackorby, C., Bossert, W., and D. Donaldson (2005): "Multi-profile Welfarism: A Generalization," Social Choice and Welfare 24, pp. 253-267.

Dworkin, R. (1981a): "What is Equality? Part 1: Equality of Welfare," Philosophy \&3 Public Affairs 10, pp. 185-246.

Dworkin, R. (1981b): "What is Equality? Part 2: Equality of Resources," Philosophy \& 8 Public Affairs 10, pp. 283-345.

Dworkin, R. (2000): Sovereign Virtue, Harvard University Press: Cambridge.

Fleurbaey, M. and A. Trannoy (2003): "The Impossibility of a Paretian Egalitarian," Social Choice and Welfare 21, pp. 243-263.

Fleurbaey, M., Tungodden, B., and H. F. Chang (2003): "Any Non-welfarist Method of Policy Assessment Violates the Pareto Principle: A Comment," Journal of Political Economy 111, pp. 1382-1385.

Foley, D. (1967): "Resource Allocation and the Public Sector," Yale Economic Essays 7, pp. 45-98.

Gotoh, R., Suzumura, K., and N. Yoshihara (2005): "Extended Social Ordering Functions for Rationalizing Fair Game Forms in the sense of Rawls and Sen," International Journal of Economic Theory 1, pp. 21-41.

Kaplow, L. and Shavell, S. (2001): "Any Non-welfarist Method of Policy Assessment Violates the Pareto Principle," Journal of Political Economy 109, pp. 281-286.

Kranich, L. (1994): "Equal Division, Efficiency, and the Sovereign Supply of Labor," American Economic Review 84, 178-189.

Mill, J. S. (1859): On Liberty, reprinted in M. Warnock (ed), Utilitarianism, London: Fontana, 1973.

Nozick, R. (1974): Anarchy, State and Utopia, Oxford: Basil Blackwell.

Pattanaik, P. K. and K. Suzumura (1996): "Individual Rights and Social Evaluation: A Conceptual Framework," Oxford Economic Papers 48, 194-212.

Pattanaik, P. K. and Xu, Y. (1990): "On Ranking Opportunity Sets in terms of Freedom of Choice," Recherches Economiques de Louvain 56, pp. 383-390.

Rawls, J. (1971): A Theory of Justice, Harvard Univ Press, Cambridge.

Roberts, K. (1980): "Interpersonal Comparability and Social Choice Theory," Review of Economic Studies 47, pp. 421-439.

Sen, A. K. (1970a): Collective Choice and Social Welfare, San Francisco: Holden-Day. Republished, Amsterdam: North-Holland, 1979.

Sen, A. K. (1970b): "The Impossibility of a Paretian Liberal," Journal of Political Economy 78, pp. 152-157. 
Sen, A. K. (1977): "On Weights and Measures: Informational Constraints in Social Welfare Analysis," Econometrica 45, pp. 1539-1572.

Sen, A. K. (1979): "Utilitarianism and Welfarism," Journal of Philosophy $\mathbf{7 6}$, pp. $463-489$.

Sen, A. K. (1980): "Equality of What ?" in S. McMurrin (ed.), Tanner Lectures on Human Values. 1, Cambridge University Press, Cambridge.

Sen, A. K. (1985): Commodities and Capabilities, North-Holland: Amsterdam.

Sen, A. K. and Williams, B. (1982): "Introduction: Utilitarianism and beyond," Sen, A. K., and Williams, B., eds, Utilitarianism and beyond, Cambridge: Cambridge University Press.

Suzumura, K. (1976): "Remarks on the Theory of Collective Choice," Economica 43, pp. 381-90.

Suzumura, K. (2004): "An Extension of Arrow's Lemma with Economic Applications," mimeo.

Tadenuma, K. (2002): "Efficiency First or Equity First? Two Principles and Rationality of Social Choice," Journal of Economic Theory 104, pp. 462-72.

Van Parijs, P. (1992): "Competing Justification of Basic Income," in van Parijs, P. (ed.) Arguing for Basic Income, Verso, London, pp. 1-43.

Van Parijs, P. (1993): Marxism Recycled, Cambridge University Press, Cambridge.

Van Parijs, P. (1995): Real Freedom for All: What (if Anything) can Justify Capitalism, Oxford University Press, Oxford.

Yamada, A. and Yoshihara, N. (2005): "Triple Implementation in Production Economies with unequal skills by Sharing Mechanisms," Institute of Economic Research Discussion Paper Series A, No. 475, Hitotsubashi University, forthcoming in International Journal of Game Theory.

Yoshihara, N. (2000): "On Efficient and Procedurally-Fair Equilibrium Allocations in Sharing Games," IER Discussion Paper No. 397, Institute of Economic Research, Hitotsubashi University.

Yoshihara, N. (2005): "A Resolution of Conflicting Claims in Constructing Social Ordering Functions," mimeo.

Yoshihara, N. (2006): "Normative Economic Foundation for the Theory of Welfare State Policies," presented at Workshop on "Real Freedom for All" with Van Parijs, Ritsumeikan University, Kyoto, July 7th, 2006.

Yoshihara, N. (2006a): "On Non-welfarist Social Ordering Functions," mimeo. 